\title{
Utilization of Stearic acid Extracted from Olive Pomace for Production of Triazoles, Thiadiazoles and Thiadiazines Derivatives of Potential Biological Activities
}

\author{
Hanaa Mohamad Soliman ${ }^{1 *}$, Amany M. Basuny ${ }^{2}$ and Shaker M. Arafat ${ }^{2}$ \\ ${ }^{1}$ Fats and Oils Dept., National Research Centre, Dokki, Giza, Egypt. \\ ${ }^{2}$ Olis \& Fats Research Dept., Food Technology Research Institute, Agricultural Research Center, Giza, Egypt.
}

\begin{abstract}
Olive Pomace was firstly dried, then pomace olive oil was extracted, and the obtained oil was hydrolyzed to produce glycerol and mixture of fatty acids ${ }^{1}$. Fatty acids mixture was separated, this mixture was then cooled, where the all saturated fatty acids were solidified, and then they were filtered off. These saturated fatty acids were identified by GC mass after esterification, and were identified as stearic, palmitic and myristic acids. Stearic acid was extracted using supercritical $\mathrm{CO}_{2}$ extractor ${ }^{2}$. The stearic acid was confirmed by means of GC mass after its esterification, and it was used as starting material for preparation of a variety of heterocyclic compounds, which were then tested for their antimicrobial activities.

Thus the long-chain fatty acid hydrazide (2) was prepared from the corresponding long-chain fatty ester with hydrazine hydrate ${ }^{3)}$. Reacting 2 with phenyl isothiocyanate afforded the corresponding thiosemicarbazide 4 . The later 4 underwent intramolecular cyclization in basic medium, and gave the $s$ triazole derivative 5, which was methylated and afforded 3-heptadecanyl-5-(methylthio)-4-phenyl-4H-1,3,4triazole (7), which was then treated with hydrazine hydrate and afforded the corresponding 1-(5-heptadecanyl-4-phenyl-4H-1,2,4-triazol-3-yl) hydrazine (8).On the other hand, thiosemicarbazide 4 underwent intramolecular cyclization in acid medium and afforded the corresponding thiadiazole derivative 6. Treatment of thiosemicarbazide 4 with ethyl chloro(arylhydrazono) acetate derivatives $9 a-b$, furnished a single product 13 (Scheme 6). Similarly, when the thiosemicarbazide 4 was treated with the phenylcarbamoylarylhydrazonyl chloride 10a-c, it afforded (3-Aryl- $N$-5-(phenylcarbamoyl)-1,3,4thiadiazol-2(3H)-ylidene)octadecanehydrazide 15a-c (Scheme 7). Also the reaction of thiosemicarbazide 4 with 2-oxo-N-arylpropanehydrazonoyl chlorides $11 \mathrm{a}-\mathrm{c}$ and $\mathrm{N}$-phenylbenzohydrazonoyl chloride $11 \mathrm{~d}$ gave the corresponding thiadiazole derivatives 16a-d as shown in Scheme 8.

A solution of thiosemicarbazide 4 was treated with the haloketones $17 \mathrm{a}-\mathrm{c}$, afforded the thiadiazine derivatives 20a-c, as shown in Scheme 9. Analogously, the thiosemicarbazide 4 was reacted with $\alpha$ haloketones 21a-b and afforded the corresponding products 22a-b (Scheme 9). The structure elucidation of all synthesized compounds is based on the elemental analysis and spectral data (IR, ${ }^{1} \mathrm{H}$ NMR, ${ }^{13} \mathrm{C}$ NMR and MS).
\end{abstract}

Key words: fatty acid hydrazides, pathological conditions, thiadiazoles, haloketones, thiosemicarbazide

\section{INTRODUCTION}

Heterocyclic compounds are well known by their pharmaceutical importance. Derivatives of triazoles, thiadiazoles and thiadiazines have played an important role in the medicinal chemistry, where they possess broad-spectrum antimicrobial activity ${ }^{4,5}$. Also they have been studied for a number of pathological conditions such as inflammation ${ }^{6,7)}$, pain $^{8-10)}$, and hypertension ${ }^{11)}$. Thus they can be used as antibacterial $^{12)}$, antimycobacterial ${ }^{13,14)}$, antifungal ${ }^{15-17)}$, antioxidant ${ }^{18)}$, and antidepressant agents ${ }^{19)}$. Seed oils have also been reported for their various biological applications such as antimicrobial ${ }^{20,21)}$, antifungal ${ }^{22)}$, Pesticidal $^{23)}$, and anticancer activities ${ }^{24)}$.

The olive pomace left over from olive oil processing which usually used as organic fertilizer, animal food and as a source for energy, was used in this paper as a source of

\footnotetext{
*Correspondence to: Hanaa Mohamad Soliman, Fats and Oils Dept., National Research Centre, Dokki, Giza, Egypt.

E-mail: chemist_hanaa@hotmail.com.

Accepted June 17, 2015 (received for review November 17, 2014)

Journal of Oleo Science ISSN 1345-8957 print / ISSN 1347-3352 online

http://www.jstage.jst.go.jp/browse/jos/ http://mc.manusriptcentral.com/jjocs
} 
stearic acid, a saturated fatty acid which has the same number of carbon atoms as oleic acid, was then appended to triazole, thiadiazole and thiadiazine nucleus, in order to combine the beneficial effects of the hetero cyclic nucleus and of the fatty acid in a new single structure of a certain biological activity on human health. Moreover these compounds are expected to have several advantages over the parent heterocyclic one, where they can easily penetrate the lipoprotein cell membrane, they may be stored in liver and skin as a lipid component that can be used whenever they needed, also the toxicity and side effects of this new compounds may be reduced by the presence of the long chain fatty acid residue which circulate the hetero nucleus.

Appended of these heterocyclic compounds to oleic acid (that was obtained from the seam source of stearic acid), along chain fatty acid with one unsaturated bond have been previously prepared, and had proved a good biological activity $^{25)}$. This work is made as to explore whether the degree of fatty acid unsaturation affect the biological activity of the heterocyclic compounds which appended to it.

The present work is aimed to utilize pomace olive as a source for stearic acid which was used as a precursor for production of novel heterocyclic compounds of potential biological activity.

\section{EXPERIMENT}

\subsection{Instruments}

All melting points were measured on a Gallenkamp electrothermal melting point apparatus. The infrared spectra were recorded for potassium bromide on a Pye Unicam Sp 3-300 and Schimadzu FT IR 8101 PC infrared spectrophotometer. The NMR spectra were recorded on a Varian Mercury VX-300 NMR spectrometer. ${ }^{1} \mathrm{H}$ spectra were run at $300 \mathrm{MHz}$. Chemical shifts are quoted in $\delta$ and were related to that of the solvents. The mass spectra were recorded on a Shimadzu GCMS-QP-1000EX mass spectrometers at 70 e.V. Elemental analyses were carried out at the Micro-analytical Center of Cairo University. Supercritical $\mathrm{CO}_{2}$ instrument used in the present study was applied Separations. Inc., Allentown, USA, model no.7071, and present in national research center, Giza, Egypt.

\subsection{Material and reagents}

Pomace olive was obtained from food technology research institute, Agriculture research center, Giza, as waste product from olive oil processing. Hydrazine hydrate, phenylhydrazine, ethyl acetoacetate, and acetoacetanilide were purchased from Aldrich Chemical Co. Acetophenone and petane-2,4-dionebromine, were purchased from Arcos Organics, Fisher Scientific Company(Belgium). Triethylamine and triphenylphosphine, were purchased from British Drug House (BDH). Phenyl isothiocyanate, sulfuryl chloride, acetonitrile, and methyl iodide were purchased from Merck Co., Germany. Sodium acetate trihydrate, sodium nitrite, anhydrous sodium sulfate, ammonium hydroxide, hydrochloric acid, sodium bicarbonate, acetic acid and sodium acetate were purchased from El-Nasr Pharmaceutical Chemical Co. (ADWIC), Egypt.

\subsection{Solvents}

Dimethylformamide (DMF), diethyl ether and benzene were purchased from Aldrich Chemical Co. Ethanol, methanol, petroleum ether and carbon tetrachloride were $\mathrm{BDH}$ reagents. Acetic acid and hexane were purchased from El-Nasr Pharmaceutical and Chemical Co. (ADWIC), Egypt.

\subsection{Pomace olive oil extraction}

Pomace olive $\left(3 \mathrm{~kg}\right.$ ) was firstly dried over night at $70^{\circ} \mathrm{C}$, and moisture was found to be $48.33 \%$, then oil was extracted by automatic Soxhlet method ${ }^{26)}$. Where dried pomace olive $(1.550 \mathrm{~kg})$ were divided to 31 sample, each one was $50 \mathrm{~g}$, A sample of $50 \mathrm{~g}$ was weighed and oil was extracted with n-hexane $(300 \mathrm{~mL})$ in a Soxhlet Apparatus at a condensation rate of 5 or 6 points per second for 4 hours at a temperature of $70^{\circ} \mathrm{C}$. The solvent was then evaporated to dryness using a rotary evaporator at $40^{\circ} \mathrm{C}^{26)}$ and the obtained olive oil was $216 \mathrm{~g}$.

\subsection{Quality parameters}

Refractive index, color index, acidity, peroxide value and UV absorption characteristics, $\mathrm{K}_{232 \mathrm{~nm}}$ (conjugated dienes) and $\mathrm{K}_{270 \mathrm{~nm}}$ (conjugated trienes) of the isolated pomace olive oil were carried out following the analytical methods described by $^{27)}$.

\subsection{Fatty acids composition}

The fatty acid of the analyzed isolated oil samples was determined by GC-Capillary column according to the methodreported by ${ }^{27)}$.

\subsection{Oil hydrolysis.}

As oil molecule is an ester derived from glycerol and three fatty acid molecules, it could be hydrolyzed with water at high temperature and pressure to produce fatty acids and glycerol as shown in Fig. 1.

Thus, oil (216 g) was hydrolyzed with distilled water (500 $\mathrm{mL}$ ) in high pressure reactor at $250^{\circ} \mathrm{C}$ and $2 \mathrm{MPa}^{1)}$, then, the reaction was left to cool to room temperature, where the reaction mixture was separated into two layers. The upper layer contained the fatty acids while the lower layer contained water and glycerol. The upper layer was then separated from the lower one, dried over anhydrous sodium sulfate and filtered off. Formation of fatty acids was confirmed by ${ }^{1} \mathrm{H}$ NMR spectrum where signal at $\delta 10.04$ was observed which indicates the presence of carboxylic hydrogen. 


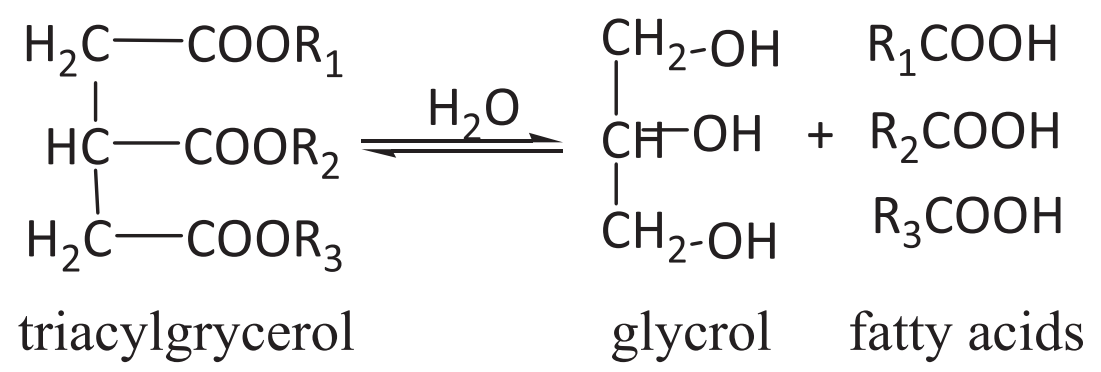

Fig. 1

The lower layer which contains water and glycerol was transferred to rotator evaporator in order to remove water, the clear color less remainder (glycerol) was found to be (22.01 g), which indicate approximately complete hydrolysis of oil.

\subsection{Stearic acid extraction using supercritical $\mathrm{CO}_{2}$ extrac- tor}

The fatty acid mixture $(188.4 \mathrm{~g})$ was cooled at $7^{\circ} \mathrm{C}$ where all saturated fatty acids were solidified, and then they were filtered off and weighted. These saturated fatty acids (35.22 g) were identified by GC mass after esterfication, and were identified as stearic acids $(13.13 \%)$, palmitic acids $(86.62 \%)$ and myristic acids $(0.26 \%)$. Stearic acid was then extracted using supercritical $\mathrm{CO}_{2}$ extractor at pressure of $40.0 \mathrm{MPa}$ and temperature of $328^{\circ} \mathrm{K}^{2}$. The obtained stearic acid was confirmed by chemical analysis (C, 76.00; $\mathrm{H}, 12.76 \%)$, milting point $\left(96.6^{\circ} \mathrm{C}\right)$ and $\mathrm{GC}$ mass after its esterfication, Then it was used as starting material for preparation of a variety of heterocyclic compounds.

Supercritical $\mathrm{CO}_{2}$ instrument can be used for the fatty acid mixture that was obtained by oil hydrolysis directly without separation of saturated fatty acids from unsaturated, but as the run is carried out only for $30 \mathrm{~g}$ sample, it was economically favorable to use the saturated fatty acid directly.

\subsection{ORGANIC PREPARATIONS}

Chloro (arylhydrazono) ethyl acetate 9a-b

General Procedure.

To a solution of ethyl 2-chloro-3-oxobutanoate (1.64 g, 10 mmol) \{prepared by dropping a solution of sulfuryl chloride $(26.8 \mathrm{~g}, 200 \mathrm{mmol})$ in dry ether $(50 \mathrm{~mL})$ over a period of $1 \mathrm{~h}$ to a cold solution of ethyl acetoacetate (23.2 g, $200 \mathrm{mmol}$ ) in dry ether $(300 \mathrm{~mL})$ with stirring. After complete addition, the reaction mixture was refluxed for $2 \mathrm{~h}$ on a water bath. The solvent was removed and the oil residue was then distilled under reduced pressure. The product was collected at $80^{\circ} \mathrm{C} / 200 \mathrm{mmHg}$ to afford $27 \mathrm{~g}(85 \%$ yield $\left.)\right\}$ in ethanol $(100 \mathrm{~mL})$, sodium acetate trihydrate $(3.0 \mathrm{~g})$ was added. After stirring for $10 \mathrm{~min}$., the mixture was cooled to $0^{\circ} \mathrm{C}$ and treated with the appropriate arene diazonium chloride solution [prepared by diazotizing the appropriate aryl- amine (10mmol) in hydrochloric acid ( $6 M, 6 \mathrm{~mL})$ with sodium nitrite solution $(0.7 \mathrm{~g}, 10 \mathrm{mmol})$ in water $(5 \mathrm{~mL})]$. The addition of the diazonium salt was carried out with rapid stirring over a period of $30 \mathrm{~min}$. The reaction mixture was stirred for further $2 \mathrm{~h}$ at $0-5^{\circ} \mathrm{C}$, and then left for $6 \mathrm{~h}$ in a refrigerator. The resulting solid was collected by filtration, washed thoroughly with water and then dried. The crude product was recrystallized from ethanol to give the corresponding hydrazonoyl chlorides $9 \mathrm{a}-\mathrm{b}^{28)}$.

Phenylcarbamoylarylhydrazonyl chlorides (10a-c)

General Procedure.

A solution of $\alpha$-chloroacetoacetanilide(2.11 g, 10 mmol) \{prepared by addition of sulfuryl chloride solution (26.8 g, $200 \mathrm{mmol})$ in dry ether $(50 \mathrm{~mL})$ over a period of $1 \mathrm{~h}$ to a cold suspension of acetoacetanilide (50g, $240 \mathrm{mmol}$ ) in $300 \mathrm{~mL}$ of dry ether, while stirring, at $0^{\circ} \mathrm{C}$. After stirring for an additional 15 min., the solvent was removed under reduced pressure and the residue was recrystallized from aqueous ethanol to afford $54.5 \mathrm{~g}$ ( $75 \%$ yields) of compound, mp. $138^{\circ} \mathrm{C}$ (lit. $\left.\left.{ }^{29)} \mathrm{mp} .137-138^{\circ} \mathrm{C}\right)\right\}$ in ethanol $(50 \mathrm{~mL})$ was stirred for $10 \mathrm{~min}$. with sodium acetate trihydrate $(1.3 \mathrm{~g}, 10$ $\mathrm{mmol})$. The mixture was then kept at $0-5^{\circ} \mathrm{C}$ and treated with cold solution of the appropriate arenediazonium chloride. The addition of the diazonium salt was carried out with rapid stirring over a period of $30 \mathrm{~min}$. The reaction mixture was stirred for further $30 \mathrm{~min}$., and then left in a refrigerator for $6 \mathrm{~h}$. The resulting solid was collected by filtration, washed thoroughly with water, and dried. The crude product was recrystallized from ethanol to afford the corresponding hydrazonyl chlorides $10 \mathrm{a}-\mathrm{c}^{30)}$.

2-Oxo-N-arylpropanehydrazonyl chlorides 11a-c

General Procedure.

To a stirred solution of 3-chloropentan-2,4-dione (1.34 g, $1 \mathrm{mmol}$ ) \{prepared by dropping of sulfuryl chloride solution ( $26.8 \mathrm{~g}, 200 \mathrm{mmol})$ in dry ether $(50 \mathrm{~mL})$ over a period of $1 \mathrm{~h}$. to a cold solution of pentane-2,4-dione (20 g, $200 \mathrm{mmol}$ ) in dry ether $(300 \mathrm{~mL})$ while stirring. After complete addition, the reaction mixture was refluxed for $2 \mathrm{~h}$ on a water bath. The solvent was removed and the oil residue was then distilled under reduced pressure. 3-Chloro-2,4-pentan-2,4-dione was collected at $49^{\circ} \mathrm{C}$ under $18 \mathrm{mmHg}$. in ethanol (100 $\mathrm{mL})$, sodium acetate trihydrate $(1.3 \mathrm{~g}, 10 \mathrm{mmol})$ was added. After stirring for $15 \mathrm{~min}$, the mixture was chilled at $0^{\circ} \mathrm{C}$ 
and treated with cold solution of the appropriate arene diazonium chloride. The addition of the diazonium salt was stirred for an additional $2 \mathrm{~h}$ at $0-5^{\circ} \mathrm{C}$, and then left for $8 \mathrm{~h}$ in a refrigerator $\left(4^{\circ} \mathrm{C}\right)$. The resulting solid was collected by filtration, washed thoroughly with water and dried. The crude product was recrystallized from ethanol to give the corresponding hydrazonoyl chlorides $11 \mathrm{a}-\mathrm{c}^{31}$.

Aryl-2-bromoethanones 17a-c.

General Procedure

To a stirred solution of acetophenone or its substituted derivatives $(100 \mathrm{mmol})$ in dry ether $(100 \mathrm{~mL})$, anhydrous aluminum chloride $(0.5 \mathrm{~g})$ was added. To the resulting mixture, bromine (16.0 g, $100 \mathrm{mmol}$ )was added dropwise over a period of $30 \mathrm{~min}$. with stirring. After complete addition, stirring was continued for further $20 \mathrm{~min}$. and the reaction mixture was poured onto cold water. The organic layer was separated, washed twice with water, then with sodium bicarbonate solution $(5 \%, 150 \mathrm{~mL})$ and finally dried over anhydrous sodium sulfate, then filtered. The solvent was removed under reduced pressure and the solid residue was recrystallized from ether to afford the corresponding compounds 17a-c $\left(\right.$ lit. $\left.^{32)}\right)$.

2-(2-phenylhydrazonno)-2-chloro-1-phenylethanone (11d)

Carbon tetrachloride ( $2 \mathrm{~mL}, 20 \mathrm{mmol}$ ) was added to a stirred suspension of 1-benzoyl-2-phenylhydrazine (4.24 g, $20 \mathrm{mmol}$ ) and triphenylphosphine (6.5 g, 25mmol) in dry acetonitrile $(40 \mathrm{~mL})$ (dried by passing through alumina and introduced directly from the column into the reaction flask). Stirring was continued for $12 \mathrm{~h}$, and then water (100 $\mathrm{mL}$ ) was added to the reaction mixture. The precipitated solid was collected by filtration, washed with water, dried and recrystallized from acetic acid to afford (1.19g, 23\%) yield of compound $11 \mathrm{~d}\left(\mathrm{mp} .128-129^{\circ} \mathrm{C}\right.$ (lit. ${ }^{33)} \mathrm{mp} .130^{\circ} \mathrm{C}$ ).

\subsection{ORGANIC SYNTHESIS AND REACTIONS}

\subsubsection{Methyl octadecanoate (Methyl stearate) (1)}

To a mixture of octadecanoic acid (stearic acid) (7.12 g, $25 \mathrm{mmol}$ ) and absolute methanol (40 mL, $1000 \mathrm{mmol})$ in dry benzene $(100 \mathrm{~mL})$, conc. sulphuric acid $(5 \mathrm{~mL})$ was added. The reaction mixture was refluxed for $6 \mathrm{~h}$. then allowed to cool. The mixture was washed with water till neutralization, then the methyl ester was separated from benzene by extraction with diethyl ether, and then the solvent was evaporated under reduced pressure.

Yield $(6.78 \mathrm{~g}, 91 \%)$, mp. $39-40^{\circ} \mathrm{C} . \mathrm{IR}(\mathrm{KBr}) \mathrm{v}_{\max } / \mathrm{cm}^{-1}$ : 2917-2847 (CH- aliphatic), $1720(\mathrm{C}=\mathrm{O}) .{ }^{1} \mathrm{H} \operatorname{NMR}\left(\mathrm{CDCl}_{3}\right)$ : $\delta 0.89(\mathrm{t}, 3 \mathrm{H}, \mathrm{J}=6.8 \mathrm{~Hz}), 1.24-1.35(\mathrm{~m}, 28 \mathrm{H}), 1.65(2 \mathrm{H}, \mathrm{tt}, \mathrm{J}$ $=6.9,7.1), 2.36(\mathrm{t}, 2 \mathrm{H}, \mathrm{J}=6.9 \mathrm{~Hz}),, 3.66\left(\mathrm{~s}, 3 \mathrm{H}, \mathrm{OCH}_{3}\right) \cdot{ }^{13} \mathrm{C}$ NMR (DMSO $\left.-\mathrm{d}_{6}\right): \delta 14.11,22.74,25.11,29.14,29.34$, 29.39, 29.70, 31.91, 33.58, 51.41, 174.1. MS $(\mathrm{m} / \mathrm{z}):\left(297 \mathrm{M}^{+}\right)$, 267, 255, 199, 143, 87, 74, 55. For $\mathrm{C}_{19} \mathrm{H}_{38} \mathrm{O}_{2}(298.29)$ : Calcd: C, 76.45 ; H, 12.83\%. Found: C, 76.43; H, 12.86\%.

\subsubsection{Octadecanyl hydrazide (2)}

Hydrazine hydrate $(0.49 \mathrm{~mL}, 10 \mathrm{mmol})$ was added to a mixture of methyl octadecanoate(1) (2.98 g, $100 \mathrm{mmol})$ in dry benzene $(30 \mathrm{~mL})$. The mixture was heated under reflux for $6 \mathrm{~h}$. then it was allowed to cool, and poured over acidified ice-cold water. The precipitated product was filtered off, washed with water, dried and recrystallized from ethanol.

Yield $(2.74 \mathrm{~g}, 92 \%)$, mp. $67-68^{\circ} \mathrm{C} . \mathrm{IR}(\mathrm{KBr}) v_{\max } / \mathrm{cm}^{-1}$ : $3330(\mathrm{NH}), 3271-3198\left(\mathrm{NH}_{2}\right), 1615(\mathrm{C}=\mathrm{O}) .{ }^{1} \mathrm{H}$ NMR $\left(\mathrm{CDCl}_{3}\right): \delta 0.90(\mathrm{t}, 3 \mathrm{H}, \mathrm{J}=6.7 \mathrm{~Hz}), 1.23-1.34(\mathrm{~m}, 28 \mathrm{H}), 1.66$ $(\mathrm{tt}, 2 \mathrm{H}, \mathrm{J}=6.9,7.1 \mathrm{~Hz}), 2.36(\mathrm{t}, 2 \mathrm{H}, \mathrm{J}=6.8 \mathrm{~Hz}), 3.51(\mathrm{~s}, 2 \mathrm{H}$, NH2), $8.89(\mathrm{~s}, 1 \mathrm{H}, \mathrm{NH}) .{ }^{13} \mathrm{C}$ NMR $\left(\mathrm{DMSO}-\mathrm{d}_{6}\right): \delta 14.12$, 22.73, 25.12, 29.12, 29.36, 29.40, 29.71, 31.90, 33.57, 176.7. MS $(\mathrm{m} / \mathrm{z}): 298\left(\mathrm{M}^{+1}\right), 297\left(\mathrm{M}^{+}\right), 239,185,129,87,55$. For $\mathrm{C}_{18} \mathrm{H}_{38} \mathrm{~N}_{2} \mathrm{O}(298.3)$ : Calcd: C, 72.42; H, 12.83; N, $9.83 \%$. Found: C, 72.45; H, 12.80; N, 9.82\%.

2.10.3 1-Octadecanoyl-4-phenylthiosemicarbazide (4)

Acid hydrazide 2(2.98 g, $10 \mathrm{mmol})$ was reacted with phenyl isothiocyanate $(1.35 \mathrm{~g}, 10 \mathrm{mmol})$ in dry dioxane (20 $\mathrm{mL}$ ) under refluxed for $6 \mathrm{~h}$., left to cool. The separated solid was filtered off, washed with ethanol, dried and recrystallized from dioxane.

Yield (3.59 g, 83\%), mp. $76-78^{\circ} \mathrm{C} . \mathrm{IR}(\mathrm{KBr}) v_{\max } / \mathrm{cm}^{-1}$ : $3491(\mathrm{NH}), 1658(\mathrm{C}=\mathrm{O}), 1343(\mathrm{C}=\mathrm{S}) .{ }^{1} \mathrm{H} \operatorname{NMR}\left(\mathrm{CDCl}_{3}\right): \delta$ $0.88(\mathrm{t}, 3 \mathrm{H}, \mathrm{J}=6.7 \mathrm{~Hz}), 1.25-1.36(\mathrm{~m}, 28 \mathrm{H}), 1.65(\mathrm{tt}, 2 \mathrm{H}, \mathrm{J}=$ 7.0, $7.2 \mathrm{~Hz}), 2.37(\mathrm{t}, 2 \mathrm{H}, \mathrm{J}=6.8 \mathrm{~Hz}), 2.54\left(\mathrm{~s}, \mathrm{D}_{2} \mathrm{O}\right.$-exchangble, $1 \mathrm{H}), 4.92\left(\mathrm{~s}, \mathrm{D}_{2} \mathrm{O}\right.$-exchangble, $\left.1 \mathrm{H}\right), 7.20-7.64(\mathrm{~m}, 5 \mathrm{H})$, $8.22\left(\mathrm{~s}, \mathrm{D}_{2} \mathrm{O}\right.$-exchangble, $\left.1 \mathrm{H}\right) .{ }^{13} \mathrm{C} \mathrm{NMR}\left(\mathrm{DMSO}-\mathrm{d}_{6}\right)$ : א14.10, 22.73, 25.12, 29.14, 29.35, 29.40, 29.70, 31.92, $33.59,124.79,126.50,129.11,134.10,137.11,137.19$, 176.62. MS (m/z):432 $\left(\mathrm{M}^{+}\right)$, 356, 255, 199, 157, 129, 97, 55. For $\mathrm{C}_{25} \mathrm{H}_{43} \mathrm{~N}_{3} \mathrm{OS}(433.31)$ Calcd: C, 69.23; H, 9.99; N, 9.69; S, $7.39 \%$.

Found: C, 69.25; H, 9.98; N, 9.71S, 7.38\%.

2.10.4 5-Heptadecanyl-4-phenyl-2H-1,2,4-triazole-3 (4H) -thione (5)

A solution of $\mathrm{KOH}(30 \mathrm{mmol})$ in water $(30 \mathrm{~mL})$ was added to the thiosemicarbazide $4(4.33 \mathrm{~g}, 10 \mathrm{mmol})$, and the mixture was refluxed for $4 \mathrm{~h}$. then left to cool and acidified with glacial acetic acid. The precipitated product was filtered off, washed with water, dried and srecrystallized from ethanol.

Yield $(3.52 \mathrm{~g}, 85 \%), \mathrm{mp} .88-89^{\circ} \mathrm{C} . \mathrm{IR}(\mathrm{KBr}) v_{\max } / \mathrm{cm}^{-1}$ : $2581(\mathrm{SH}), 1645(\mathrm{C}=\mathrm{N}), 1345(\mathrm{C}=\mathrm{S}) .{ }^{1} \mathrm{H} \operatorname{NMR}\left(\mathrm{CDCl}_{3}\right): \delta$ $0.90(\mathrm{t}, 3 \mathrm{H}, \mathrm{J}=6.6 \mathrm{~Hz}), 1.25-1.36(\mathrm{~m}, 28 \mathrm{H}), 1.66(\mathrm{tt}, 2 \mathrm{H}, \mathrm{J}=$ $6.9,7.2, \mathrm{~Hz}), 2.36(\mathrm{t}, 2 \mathrm{H}, \mathrm{J}=7.0 \mathrm{~Hz}), 7.21-7.64(\mathrm{~m}, 5 \mathrm{H})$, $8.04\left(\mathrm{~s}, \mathrm{D}_{2} \mathrm{O}\right.$-exchangble, $\left.1 \mathrm{H}\right) .{ }^{13} \mathrm{C} \mathrm{NMR}\left(\mathrm{DMSO}-\mathrm{d}_{6}\right)$ : $\delta 14.09,22.72,25.41,29.15,29.35,29.38,29.70,31.33$, $31.89,128.20,128.82,129.20,129.97,153.11,168.90 . \mathrm{MS}$ $(\mathrm{m} / \mathrm{z}): 415\left(\mathrm{M}^{+1}\right), 414\left(\mathrm{M}^{+}\right), 279,241,185,171,77,57$. For $\mathrm{C}_{25} \mathrm{H}_{41} \mathrm{~N}_{3} \mathrm{~S}$ (415.3): Calcd: C, 72.24; H, 9.94; N, 10.11; S, $7.71 \%$. Found: C, 72.26; H, 9.93; N, 10.13; S, 7.70\%. 


\subsubsection{3-Heptadecanyl-5-(methylthio) -4-phenyl-4H-1,3,4- triazole (6)}

Fused sodium acetate $(0.2 \mathrm{~g})$ and methyl iodide $(1.42 \mathrm{~g}$, $10 \mathrm{mmol}$ ) were added to a suspension of 1,2,4 triazol-5-thiol $5(4.15 \mathrm{~g}, 10 \mathrm{mmol})$ in $20 \mathrm{~mL}$ ethanol,. The mixture was refluxed for $9 \mathrm{~h}$., then left to cool and poured over crushed ice and kept overnight in a refrigerator. The formed solid was filtered off, washed with ethanol, dried and recrystallized from ethanol.

Yield $(3.52 \mathrm{~g}, 82 \%), \mathrm{mp} .121-122^{\circ} \mathrm{C} \cdot \mathrm{IR}(\mathrm{KBr}) v_{\max } / \mathrm{cm}^{-1}$ : 1623-1602 $(2 \mathrm{C}=\mathrm{N}), 1590(\mathrm{C}=\mathrm{C}) .{ }^{1} \mathrm{H} \mathrm{NMR}\left(\mathrm{CDCl}_{3}\right): \delta 0.92$ $(\mathrm{t}, 3 \mathrm{H}, \mathrm{J}=7.0 \mathrm{~Hz}), 1.54-1.33(\mathrm{~m}, 28 \mathrm{H}), 1.64(\mathrm{tt}, 2 \mathrm{H}, \mathrm{J}=6.9$, $7.1 \mathrm{~Hz}), 2.55(\mathrm{t}, 2 \mathrm{H}, \mathrm{J}=6.8 \mathrm{~Hz}), 3.44(\mathrm{~s}, 3 \mathrm{H}), 7.21-7.55(\mathrm{~m}$, $5 \mathrm{H}) .{ }^{13} \mathrm{C}$ NMR (DMSO $\left.-\mathrm{d}_{6}\right): \delta 14.12,14.93,22.78,29.28$, $25.42,29.35,29.60,29.70,31.27,31.90,128.21,128.82$, $129.23,129.9,147.52,153.32 . \mathrm{MS}(\mathrm{m} / \mathrm{z}): 428\left(\mathrm{M}^{+}\right), 382$, 342, 241, 199, 77, 55. For $\mathrm{C}_{26} \mathrm{H}_{43} \mathrm{~N}_{3} \mathrm{~S}$ (429.7) Calcd.: C, 72.67 : H, 10.09; N, 9.78; S, 7.26\%. Found : C, 72.66; H, 10.10; N, $9.75 ; \mathrm{S}, 7.28 \%$.

2.10.6 5-Heptadecanyl-4-phenyl-4H-1,2,4-triazol-3-yl) hydrazine (7)

A suspension of 3-heptadecanyl-5-(methylthio) -4-phenyl-4H-1,3,4-triazole (6) (2.14 g, $5 \mathrm{mmol})$ in ethanol (20 $\mathrm{mL})$, was added to hydrazine hydrate $(0.245 \mathrm{~g}, 5 \mathrm{mmol})$, and refluxed for $8 \mathrm{~h}$. The mixture was cooled, poured over acidified ice. The formed precipitated was filtered off, washed with ethanol, dried and recrystallized from ethanol.

Yield $(1.81 \mathrm{~g}, 88 \%), \mathrm{mp} \cdot 129-130^{\circ} \mathrm{C} \cdot \mathrm{IR}(\mathrm{KBr}) v_{\max } / \mathrm{cm}^{-1}$ : $3320(\mathrm{NH}), 3220-3197\left(\mathrm{NH}_{2}\right), 1637-1622(2 \mathrm{C}=\mathrm{N}) .{ }^{1} \mathrm{H}$ NMR $\left(\mathrm{CDCl}_{3}\right): \delta 0.89(\mathrm{t}, 3 \mathrm{H}, \mathrm{J}=6.9 \mathrm{~Hz}), 1.24-1.33(\mathrm{~m}, 28 \mathrm{H}), 1.64$ $(\mathrm{tt}, 2 \mathrm{H}, \mathrm{J}=6.8,7.0 \mathrm{~Hz}), 2.56(\mathrm{t}, 2 \mathrm{H}, \mathrm{J}=6.9 \mathrm{~Hz}), 2.70(\mathrm{~s}$, $\mathrm{D}_{2} \mathrm{O}$-exchangble, $\left.2 \mathrm{H}, \mathrm{NH}_{2}\right)$, 4. $13\left(\mathrm{~s}, \mathrm{D}_{2} \mathrm{O}\right.$-exchangble, $1 \mathrm{H}$, NH) , 7.22-7.64 (m, 5H) ${ }^{13} \mathrm{C}$ NMR (DMSO $\left.-\mathrm{d}_{6}\right): \delta 14.11$, $22.77,25.77,29.30,29.35,29.59,29.71,31.31,31.89$, $128.23,128.80,129.27,129.9,148.88,153.21 . \mathrm{MS}(\mathrm{m} / \mathrm{z})$ : $412\left(\mathrm{M}^{+}\right), 336,365,241,171,87,77,55$. For $\mathrm{C}_{25} \mathrm{H}_{41} \mathrm{~N}_{5}$ (413.35) Calcd: C, 72.59; H, 10.48; N, 16.93\%. Found: C, $72.60 ; \mathrm{H}, 10.47$; N, $16.92 \%$.

2.10.7 5-Heptadecanyl- $N$-phenyl-1,3,4-thiadiazole-2-amine (8)

The phenylthiosemicarbazide $4(4.33 \mathrm{~g}, 10 \mathrm{mmol})$ was treated with sulphuric acid $(98 \%, 10 \mathrm{~mL})$ and refluxed for $12 \mathrm{~h}$. then allowed to cool, and poured over crushed ice. The mixture was neutralized with ammonia solution. The precipitated product was then filtered off, washed with water, dried and recrystallized from ethanol.

Yield $(3.32 \mathrm{~g}, 80 \%), \mathrm{mp} .102-103^{\circ} \mathrm{C} \cdot \mathrm{IR}(\mathrm{KBr}) v_{\max } / \mathrm{cm}^{-1}$ : $3595(\mathrm{NH}), 1623,1605(2 \mathrm{C}=\mathrm{N}) .{ }^{1} \mathrm{H} \mathrm{NMR}\left(\mathrm{CDCl}_{3}\right): 0.91(\mathrm{t}$, $3 \mathrm{H}, \mathrm{J}=6.7 \mathrm{~Hz}), 1.28-1.33(\mathrm{~m}, 28 \mathrm{H}), 1.65(\mathrm{tt}, 2 \mathrm{H}, \mathrm{J}=6.9,7.1$ $\mathrm{Hz}), 2.56(\mathrm{t}, 2 \mathrm{H}, \mathrm{J}=6.9 \mathrm{~Hz}), 9.73\left(\mathrm{~s}, \mathrm{D}_{2} \mathrm{O}\right.$-exchangble, $1 \mathrm{H}$, $\mathrm{NH}) .{ }^{13} \mathrm{C}$ NMR $\left(\mathrm{DMSO}-\mathrm{d}_{6}\right): \delta 14.13,22.79,28.58,29.34$, $29.59,29.69,29.29,31.11,31.91,152.68,168.49 . \mathrm{MS}(\mathrm{m} /$ z): $414\left(\mathrm{M}^{+}\right), 279,255,213,185,111,87,77,55$. For $\mathrm{C}_{25} \mathrm{H}_{41} \mathrm{~N}_{3} \mathrm{~S}$ (415.3) Calcd: C, 72.24; H, 9.94; N, 10.11; S,
7.71\%. Found: C, 72.23; H, 9.93; N, 10.13; S, 7.69\%.

2.10.8 Ethyl-4-aryl-4,5-dihydro (-5-octadecaneoylimino) -1,3,4-thiadiazole-2-carboxylates $13 a-b$

General procedure:

A solution of thethiosemicarbazide $4(4.33 \mathrm{~g}, 10 \mathrm{mmol})$ in dimethylformamide $(20 \mathrm{~mL})$, and triethylamine $(0.5 \mathrm{~mL})$, was portion wisely added to the appropriate ethyl chloro (arylhydrazono) acetate $\mathbf{9}(10 \mathrm{mmol})$ over a period of 30 min. After the addition was completed, the reaction mixture was stirred at room temperature for $10 \mathrm{~h}$. during which hydrazonoyl halide was dissolved and the product was precipitated. Then the formed solid product was filtered off, washed with water, dried and recrystallized from ethanol and afford the corresponding thiadiazole derivatives 13a-b.

2.10.9 Ethyl-4,5-dihydro-5- (octadecanoylimino) -4(4-chlorophenyl)-1,3,4-thiadiazole-2-carboxylate (13a)

Yield $\left(3.83\right.$ g, 68\% ), mp. $114-115^{\circ} \mathrm{C} . \mathrm{IR}(\mathrm{KBr}) v_{\max } / \mathrm{cm}^{-1}$ : $3365(\mathrm{NH}), 1746-1667(2 \mathrm{C}=\mathrm{O}), 1627-1594(2 \mathrm{C}=\mathrm{N}) .{ }^{1} \mathrm{H}$ $\operatorname{NMR}\left(\mathrm{CDCl}_{3}\right): \delta 0.91(\mathrm{t}, 3 \mathrm{H}, \mathrm{J}=7.0 \mathrm{~Hz}), 1.27-1.33(\mathrm{~m}$, $28 \mathrm{H}), 1.37(\mathrm{t}, 3 \mathrm{H}, \mathrm{J}=7.13 \mathrm{~Hz}), 1.59,(\mathrm{tt}, 2 \mathrm{H}, \mathrm{J}=6.9,7.1$ $\mathrm{Hz}), 2.24(\mathrm{t}, 2 \mathrm{H}, \mathrm{J}=6.8 \mathrm{~Hz}), 3.75(\mathrm{q}, 2 \mathrm{H}, \mathrm{J}=7.13 \mathrm{~Hz})$, 7.1-7.3(m, $4 \mathrm{H}, \mathrm{Ar}), 8.00\left(\mathrm{~s}, \mathrm{D}_{2} \mathrm{O}\right.$-exchangble, $\left.1 \mathrm{H}, \mathrm{NH}\right) .{ }^{13} \mathrm{C}$ NMR (DMSO $\left.-\mathrm{d}_{6}\right): \delta 13.82,14.10,22.77,26.01,29.30$, 29.34, 29.39, 29.69, 31.92, 36.58, 60.71, 117.72, 124.32, 129.71, 144.62, 154.02, 156.21, 161.8, 167.50. MS (m/z): $564\left(\mathrm{M}^{+1}\right), 563\left(\mathrm{M}^{+}\right), 453,267,2213,157,161,87,55$. For $\mathrm{C}_{29} \mathrm{H}_{44} \mathrm{ClN}_{4} \mathrm{O}_{3} \mathrm{~S}$ (564.29) Calcd: C, 61.62; H, 8.02; Cl, 6.27; N, 9.91; S, 5.67\%. Found: C, 61.61; H, 7.03; Cl, 6.28; N, 9.90; S, $5.65 \%$.

2.10.10 Ethyl-4,5-dihydro-5- (octadecanoylimino)-4- ( $p$ tolyl)-1,3,4-thiadiazole-2-carboxylate (13b)

Yield $(3.86 \mathrm{~g}, 71 \%)$, mp. $124-125^{\circ} \mathrm{C} . \mathrm{IR}(\mathrm{KBr}) v_{\max } / \mathrm{cm}^{-1}$ : $3330(\mathrm{NH}), 1737-1684(2 \mathrm{C}=\mathrm{O})$. MS $(\mathrm{m} / \mathrm{z}): 544\left(\mathrm{M}^{+}\right), 425$, 269, 132, 119, 87, 55. For $\mathrm{C}_{30} \mathrm{H}_{48} \mathrm{~N}_{4} \mathrm{O}_{3} \mathrm{~S}(544.345)$ Calcd: C, $66.14 ; \mathrm{H}, 8.88 ; \mathrm{N}, 10.28 ; \mathrm{S}, 5.89 \%$. Found: C, 66.12; H, 8.89; N, 10.30; S, 5.87\%.

2.10.11 3-Aryl-N-5- (phenylcarbamoyl) -1,3,4-thiadiazol-2 (3Hylidene) octadecanehydrazide (15a-c)

General procedure:

To a stirred solution of the thiosemicarbazide 4(4.33 g, $10 \mathrm{mmol})$ in dimethylformamide $(20 \mathrm{~mL})$, and triethylamine $(0.5 \mathrm{~mL})$, the appropriate phenylcarbamoylarylhydrazonyl chloride 10 ( $0.10 \mathrm{mmol})$ was added portion wise over a period of $30 \mathrm{~min}$. After the addition was completed, the reaction mixtures were stirred for $10 \mathrm{~h}$. during which hydrazonoyl halide went in to solution and a new solid product was formed. The solid product was filtered off, washed with water, dried and recrystallized from the ethanol to afford the corresponding thiadiazole derivatives 15a-c.

2.10.12 3-Phenyl-5- $N$ - (phenylcarbomyl)-1,3,4-thiadiazol-2 $(3 H)$-ylidene) octadecanehydrazide (15a)

Yield $(3.86 \mathrm{~g}, 67 \%), \mathrm{mp} .116-117^{\circ} \mathrm{C} . \mathrm{IR}(\mathrm{KBr}) v_{\max } / \mathrm{cm}^{-1}$ : 
$3334(\mathrm{NH}), 1736,1663(2 \mathrm{C}=\mathrm{O}) .{ }^{1} \mathrm{H} \operatorname{NMR}\left(\mathrm{CDCl}_{3}\right): \delta 0.90$ (t, 3H, J = 7.0 Hz), 1.25-1.35(m, 28H), $1.64(\mathrm{tt}, 2 \mathrm{H}, \mathrm{J}=6.8$, $7.0, \mathrm{~Hz}), 2.36(\mathrm{t}, 2 \mathrm{H}, \mathrm{J}=6.8 \mathrm{~Hz}), 6.47-7.65(\mathrm{~m}, 10 \mathrm{H}, \mathrm{Ar})$, 8.00 (s, D2O-exchangble, $1 \mathrm{H}, \mathrm{NH}) \cdot{ }^{13} \mathrm{C}$ NMR (DMSO $-\mathrm{d}_{6}$ ): $\delta 14.10,22.73,25.81,29.35,29.39,29.65,29.70,31.90$, $37.58,116.55,121.62,124.33,124.40,129.00,129.82$, 136.21, 146.42, 154.03, 156.23, 161.22, 168.52. MS $(\mathrm{m} / \mathrm{z})$ : $576\left(\mathrm{M}^{+}\right), 472,296,179,105,87,77,55$. For $\mathrm{C}_{33} \mathrm{H}_{47} \mathrm{~N}_{5} \mathrm{O}_{2} \mathrm{~S}$ (577.345) Calcd: C, 68.59; H, 8.20; N, 12.12; S, 5.55\%. Found: C, 68.60; H, 7.18; N, 12.13; S, 5.54\%.

2.10 .13 3-(4-Chlorophenyl)-5- $N$ - (phenylcarbomyl) -1,3,4-thiadiazol-2 (3H) -ylidene) octadecane hydrazide $(15 b)$

Yield $(4.21 \mathrm{~g}, 69 \%), \mathrm{mp} .122-123^{\circ} \mathrm{C} \cdot \mathrm{IR}(\mathrm{KBr}) v_{\max } / \mathrm{cm}^{-1}$ : $3329(\mathrm{NH}), 1740,1661(2 \mathrm{C}=\mathrm{O})$. MS $(\mathrm{m} / \mathrm{z}): 610\left(\mathrm{M}^{+}\right), 472$, 341, 255, 134, 87, 77, 55. For $\mathrm{C}_{33} \mathrm{H}_{46} \mathrm{ClN}_{5} \mathrm{O}_{2} \mathrm{~S}(611.306)$ Calcd: C, 64.74; H, 7.57; Cl, 5.79; N, 11.44; S, 5.24\%. Found: C, $65.73 ; \mathrm{H}, 7.59 ; \mathrm{Cl}, 5.78 ; \mathrm{N}, 11.45 ; \mathrm{S}, 5.23 \%$.

2.10.14 (5- $N$-(Phenylcarbomyl) -3- ( $p$-tolyl) -1,3,4-thiadiazol-2 (3H) -ylidene) octadecanehydrazide (15c)

Yield $(3.96 \mathrm{~g}, 67 \%), \mathrm{mp} .126-127^{\circ} \mathrm{C} . \mathrm{IR}(\mathrm{KBr}) v_{\max } / \mathrm{cm}^{-1}$ : $3329(\mathrm{NH}), 1747,1661(2 \mathrm{C}=\mathrm{O}) .{ }^{1} \mathrm{H} \mathrm{NMR}\left(\mathrm{CDCl}_{3}\right): \delta 0.88$ $(\mathrm{t}, 3 \mathrm{H}, \mathrm{J}=6.8 \mathrm{~Hz}), 1.26-1.33(\mathrm{~m}, 28 \mathrm{H}), 1.62(\mathrm{tt}, 2 \mathrm{H}, \mathrm{J}=6.8$, $7.0, \mathrm{~Hz}), 2.32(\mathrm{t}, 2 \mathrm{H}, \mathrm{J}=6.9 \mathrm{~Hz}), 2.45(\mathrm{~s}, 3 \mathrm{H}), 6.44-7.6(\mathrm{~m}, 9$ $\mathrm{H}, \mathrm{Ar}), 8.03\left(\mathrm{~s}, \mathrm{D}_{2} \mathrm{O}\right.$-exchangble, $\left.1 \mathrm{H}, \mathrm{NH}\right) . \mathrm{MS}(\mathrm{m} / \mathrm{z}): 590(\mathrm{M}$ +) $, 472,239,134,119,87,77,55$. For $\mathrm{C}_{34} \mathrm{H}_{49} \mathrm{~N}_{5} \mathrm{O}_{2} \mathrm{~S}$ (591.361) Calcd: C, 69.00; H, 8.34; N, 11.83; S, 5.42\%. Found: C, 69.02; H, 8.31; N, 11.85; S, 5.41\%.

2.10.15 N-(3-Aryl-5-substituted-1,3,4-triazol-2 (3H)-ylidene) octadecanehydrazide 16a-d

General procedure:

To a stirred solution of the thiosemicarbazide 4(4.33 g, $10 \mathrm{mmol})$ in dimethylformamide $(20 \mathrm{~mL})$, and triethylamine $(0.5 \mathrm{~mL})$, the appropriate hydrazonoyl chloride 11 (10 mmol) was added portion wise over a period of $30 \mathrm{~min}$. After the addition was completed, the reaction mixture was stirred for $12 \mathrm{~h}$. during which hydrazonoyl halide went in to solution and the new solid product was filtered off, washed with water, dried and recrystallized from the ethanol to afford the corresponding thiadiazole derivatives 16a-d.

2.10.16 N-(5-Acetyl-3-phenyl-1,3,4-triazol-2 (3H)-ylidene) octadecanehydrazide (16a)

Yield $(3.65 \mathrm{~g}, 73 \%), \mathrm{mp} .83-84^{\circ} \mathrm{C} . \mathrm{IR}(\mathrm{KBr}) v_{\text {max }} / \mathrm{cm}^{-1}$ : $3371(\mathrm{NH}), 1740,1666(2 \mathrm{C}=\mathrm{O}), 1588(\mathrm{C}=\mathrm{N}) .{ }^{1} \mathrm{H}$ NMR $\left(\mathrm{CDCl}_{3}\right): \delta 0.89(\mathrm{t}, 3 \mathrm{H}, \mathrm{J}=6.8 \mathrm{~Hz}), 1.27-1.33(\mathrm{~m}, 28 \mathrm{H}), 1.59$ $(\mathrm{tt}, 2 \mathrm{H}, \mathrm{J}=6.9,7.1 \mathrm{~Hz}), 1.98(\mathrm{~s}, 3 \mathrm{H}), 2.34(\mathrm{t}, 2 \mathrm{H}, \mathrm{J}=6.8$ $\mathrm{Hz}), 7.1-7.4(\mathrm{~m}, 5 \mathrm{H}), 7.89\left(\mathrm{~s}, \mathrm{D}_{2} \mathrm{O}\right.$-exchangble, $\left.1 \mathrm{H}\right) \cdot{ }^{13} \mathrm{C}$ $\operatorname{NMR}\left(\right.$ DMSO $\left.-\mathrm{d}_{6}\right): \delta 14.9,22.77,23.70,25.71,29.24,29.39$, 29.40, 29.73, 31.91, 38.38, 116.50, 120.22, 129.81, 149.00, 155.32, 156.19, 167.7, 191.80. MS $(\mathrm{m} / \mathrm{z}): 499\left(\mathrm{M}^{+}\right), 500\left(\mathrm{M}^{+1}\right)$, 395, 241, 199, 111, 87, 77. For $\mathrm{C}_{28} \mathrm{H}_{44} \mathrm{~N}_{4} \mathrm{O}_{2} \mathrm{~S}(500.74)$ Calcd: C, 67.16 ; H, 8.86; N, 11.19; S, 6.40\%. Found: C, 67.18; H, 8.84; N, 11.18; S, 6.41\%.
2.10.17 N-(5-Acetyl-3-(3-bromophenyl)-1,3,4-triazol-2 (3H)-ylidene) octadecanehydrazide (16b)

Yield $(3.81 \mathrm{~g}, 66 \%), \mathrm{mp} .85-86^{\circ} \mathrm{C} . \mathrm{IR}(\mathrm{KBr}) v_{\max } / \mathrm{cm}^{-1}$ : $3380(\mathrm{NH}), 1740,1671(2 \mathrm{C}=\mathrm{O}), 1590(\mathrm{C}=\mathrm{N}) . \mathrm{MS}(\mathrm{m} / \mathbf{z})$ : $577\left(\mathrm{M}^{+}\right), 423,336,255,154,199,87,77,55$. For $\mathrm{C}_{28} \mathrm{H}_{43} \mathrm{Br}$ $\mathrm{N}_{4} \mathrm{O}_{2} \mathrm{~S}(578.229)$ Calcd: C, 58.02; H, 7.48; Br, 13.79; N, 9.67; S, 5.52\%. Found: C, 58.03; H, 7.46; Br, 13.81; N, 9.66; S, $5.51 \%$.

2.10.18 N-(5-Acetyl-3- (4-tolyl)-1,3,4-triazol-2 (3H) -ylidene) octadecanehydrazide (16c)

Yield $(3.59 \mathrm{~g}, 70 \%), \mathrm{mp} .80-81^{\circ} \mathrm{C} . \mathrm{IR}(\mathrm{KBr}) v_{\max } / \mathrm{cm}^{-1}$ : $3370(\mathrm{NH}), 1741,1661(2 \mathrm{C}=\mathrm{O}), 1587(\mathrm{C}=\mathrm{N}) \cdot \mathrm{MS}(\mathrm{m} / \mathrm{z})$ : $514\left(\mathrm{M}^{+1}\right), 513\left(\mathrm{M}^{+}\right), 423,354,267,185,143,87,55$. For $\mathrm{C}_{29} \mathrm{H}_{46} \mathrm{~N}_{4} \mathrm{O}_{2} \mathrm{~S}(514.33)$ Calcd: C, 67.66; H, 9.01; N, 10.88; S, $6.23 \%$. Found: C, $67.65 ; \mathrm{H}, 9.03 ; \mathrm{N}, 10.86 ; \mathrm{S}, 6.24 \%$.

2.10.19 N-(3-Phenyl-5-benzoyl-1,3,4-thiadiazol-2 (3H) -ylidene) octadecanehydrazide (16d)

Yield $(3.87 \mathrm{~g}, 69 \%), \mathrm{mp} .119-120^{\circ} \mathrm{C} . \mathrm{IR}(\mathrm{KBr}) v_{\max } / \mathrm{cm}^{-1}$ : $3340(\mathrm{NH}), 1735,1680(2 \mathrm{C}=\mathrm{O}) \cdot{ }^{13} \mathrm{C}$ NMR $\left(\mathrm{DMSO}-\mathrm{d}_{6}\right)$ : 814.11, 22.80, 26.01, 29.30, 29.34, 29.39, 29.69, 31.92, 36.58, 116.1, 118.9, 129.6, 129.3, 129.9, 134.61, 137.90, 146.5, 153.92, 156.41, 161.8, 168.4. MS $(\mathrm{m} / \mathrm{z}): 562\left(\mathrm{M}^{+1}\right)$, $561\left(\mathrm{M}^{+}\right), 485,380,340,241,129,87,77,55$. For $\mathrm{C}_{33} \mathrm{H}_{46} \mathrm{~N}_{4} \mathrm{O}_{2} \mathrm{~S}(562.33)$ Calcd: C, 70.42; H, 8.24; N, 9.95; S, $5.70 \%$. Found: C, 70.43; H, 8.22; N, 9.96; S, 5.72\%.

2.10.20 (5-Heptadecanyl)-2-phenylamino-4H-1,3,4-thiadiazin-6-yl) (Aryl) methan- one 20a-c

General procedure:

The appropriate a-haloketones $17(10 \mathrm{mmol})$ and triethylamine $(0.5 \mathrm{~mL})$ were added to a steered solution of the thiosemicarbazide 4(4.33 g, $10 \mathrm{mmol})$ in ethanol $(20 \mathrm{~mL})$ and the mixture was heated under reflux for $6 \mathrm{~h}$. The solvent was evaporated under reduced pressure, and the formed solid was filtered off, washed with ethanol, dried and recrystallized from DMF to afford the corresponding products 20a-c. The physical and spectral data of the synthesized compounds are listed below.

2.10.21 (5-Heptadecanyl-2-phenylamino-4H-1,3,4-thiadiazin-6-yl) (phenyl) methanone (20a)

Yield $(3.67 \mathrm{~g}, 69 \%), \mathrm{mp} .90-91^{\circ} \mathrm{C} . \mathrm{IR}(\mathrm{KBr}) v_{\max } / \mathrm{cm}^{-1}$ : 3384, $3312(2 \mathrm{NH}), 1637(\mathrm{C}=\mathrm{O}) .{ }^{1} \mathrm{H} \mathrm{NMR}\left(\mathrm{CDCl}_{3}\right): \delta 0.91$ $(\mathrm{t}, 3 \mathrm{H}, \mathrm{J}=6.7 \mathrm{~Hz}), 1.29-1.35(\mathrm{~m}, 28 \mathrm{H}), 1.65(\mathrm{tt}, 2 \mathrm{H}, \mathrm{J}=6.9$, $7.1 \mathrm{~Hz}), 2.39(\mathrm{t}, 2 \mathrm{H}, \mathrm{J}=6.9 \mathrm{~Hz}), 4.42\left(\mathrm{~s}, \mathrm{D}_{2} \mathrm{O}\right.$-exchangble, $1 \mathrm{H}), 7.27-7.63(\mathrm{~m}, 10 \mathrm{H}), 8.81\left(\mathrm{~s}, \mathrm{D}_{2} \mathrm{O}\right.$-exchangble, $\left.1 \mathrm{H}\right) .{ }^{13} \mathrm{C}$ NMR $\left(\right.$ DMSO $\left.-\mathrm{d}_{6}\right): \delta 14.11,22.79,24.71,25.58,29.14$, $29.34,29.39,29.71,31.88,99.01,116.5,118.8,129.33$, $129.60,129.94,134.64,137.92,144.6,154.85,171.00$, 188.6. MS $(\mathrm{m} / \mathrm{z}): 532\left(\mathrm{M}^{+}\right), 441,400,252,241,143,87,77$, 55. For $\mathrm{C}_{33} \mathrm{H}_{47} \mathrm{~N}_{3} \mathrm{OS}(533.34)$ Calcd: C, $74.25 ; \mathrm{H}, 8.87 ; \mathrm{N}$, 7.87 ; S, $6.01 \%$. Found: C, 74.24; H, 8.89; N, 7.85; S, $6.02 \%$. 2.10.22 (4-(Chlorophenyl)-5-heptadecanyl-2-phenylamino-4H-1,3,4-thiadiazin-6-yl) (phenyl) methanone (20b)

Yield $(4.02 \mathrm{~g}, 71 \%), \mathrm{mp} .88-89^{\circ} \mathrm{C} . \mathrm{IR}(\mathrm{KBr}) v_{\max } / \mathrm{cm}^{-1}$ : 
3319, $3289(2 \mathrm{NH}), 1743(\mathrm{C}=\mathrm{O}) . \mathbf{M S}(\mathrm{m} / \mathbf{z}): 567\left(\mathrm{M}^{+1}\right), 566$ $\left(\mathrm{M}^{+}\right), 475,434,296,143,87,77,55$. For $\mathrm{C}_{33} \mathrm{H}_{46} \mathrm{ClN}_{3} \mathrm{OS}$ (567.31) Calcd: C, 69.75; H, 8.16; Cl, 6.24; N, 7.39; S, $5.64 \%$. Found: C, $69.76 ; \mathrm{H}, 7.15 ; \mathrm{Cl}, 6.23 ; \mathrm{N}, 7.38 ; \mathrm{S}$, $5.65 \%$.

2.10.23 5-Heptadecanyl-2-phenylamino-4H-1,3,4-thiadiazin-6-yl) (p-tolyl) methanone (20c)

Yield $(3.88 \mathrm{~g} 71 \%), \mathrm{mp} .85-86^{\circ} \mathrm{C} . \mathrm{IR}(\mathrm{KBr}) v_{\max } / \mathrm{cm}^{-1}$ : $3315,3288(2 \mathrm{NH}), 1630(\mathrm{C}=\mathrm{O})$. MS $(\mathrm{m} / \mathrm{z}): 547\left(\mathrm{M}^{+1}\right), 546$ $\left(\mathrm{M}^{+}\right), 470,414,252,129,87,77$. For $\mathrm{C}_{34} \mathrm{H}_{49} \mathrm{~N}_{3} \mathrm{OS}(547.36)$ Calcd: C, $74.54 ; \mathrm{H}, 9.02 ; \mathrm{N}, 7.67 ; \mathrm{S}, 5.58 \%$. Found: C, $74.55 ; \mathrm{H}, 9.00 ; \mathrm{N}, 7.68 ; \mathrm{S}, 5.57 \%$.

2.10.24 (5-Alkyl-2- (phenylamino) -4H-1,3,4-thiadiazin6-yl) octadecan-1-one 22a-b

General procedure

The appropriate a-haloketone $21(10 \mathrm{mmol})$ and triethylamine $(0.5 \mathrm{~mL})$ were added to a solution of the thiosemicarbazide $4(4.31 \mathrm{~g}, 10 \mathrm{mmol})$ in ethanol $(20 \mathrm{~mL})$. The mixture was heated under reflux for $4 \mathrm{~h}$. The solvent was evaporated under reduced pressure, and the formed solids were washed from ethanol, dried and recrystallized from ethanol to afford the corresponding products 22a-b. The physical and spectral data of the synthesized compounds are listed below.

2.10.25 1-(5-(Heptadecanyl)-2-(phenylamino) -4H-1,3,4thiadiazin-6-yl) ethanone (22a)

Yield $(3.39 \mathrm{~g}, 72 \%), \mathrm{mp} .79-80^{\circ} \mathrm{C} . \mathrm{IR}(\mathrm{KBr}) v_{\max } / \mathrm{cm}^{-1}$ : $3410,3380(2 \mathrm{NH}), 1737(\mathrm{C}=\mathrm{O}) .{ }^{1} \mathrm{H} \mathrm{NMR}\left(\mathrm{CDCl}_{3}\right): \delta 0.90$ $(\mathrm{t}, 3 \mathrm{H}, \mathrm{J}=6.8 \mathrm{~Hz}), 1.26-1.34(\mathrm{~m}, 28 \mathrm{H}), 1.36(\mathrm{tt}, 2 \mathrm{H}, \mathrm{J}=6.8$, $7.1 \mathrm{~Hz}), 2.14(\mathrm{t}, 2 \mathrm{H}, \mathrm{J}=6.9 \mathrm{~Hz}), 2.36\left(\mathrm{~s}, 3 \mathrm{H}, \mathrm{CH}_{3}\right), 4.02(\mathrm{~s}$, $\mathrm{D}_{2} \mathrm{O}$-exchangble, $\left.1 \mathrm{H}, \mathrm{NH}\right), 6.62-7.01(\mathrm{~m}, 5 \mathrm{H}), 7.83\left(\mathrm{~s}, \mathrm{D}_{2} \mathrm{O}-\right.$ exchangble, $1 \mathrm{H}, \mathrm{NH}) \cdot{ }^{13} \mathrm{C}$ NMR $\left(\right.$ DMSO $\left.-\mathrm{d}_{6}\right): \delta 14.11$, 22.71, 24.46, 25.81, 26.25, 29.32, 29.36, 29.69, 29.73, 31.89, $101.32,116.4,118.7,129.5,144.6,154.00,166.21,192.62$. MS $(\mathrm{m} / \mathrm{z}): 470\left(\mathrm{M}^{+}\right), 379,351,252,102,111,92,87,77,55$. For $\mathrm{C}_{28} \mathrm{H}_{45} \mathrm{~N}_{3} \mathrm{OS}(471.33)$ Calcd: C, 71.29; H, 9.61; N, 8.91; S, $6.80 \%$. Found: C, 71.30; H, 9.60; N, 8.90; S, 6. 82\%.

2.10.26 Ethyl-5-(heptadec-8-enyl)-2-(phenylamino)-4H1,3,4-thiadiazine-6-carboxylate (22b)

Yield $(3.5 \mathrm{~g}, 71 \%), \mathrm{mp} \cdot 82-83^{\circ} \mathrm{C} \cdot \mathrm{IR}(\mathrm{KBr}) v_{\max } / \mathrm{cm}^{-1}$ : $3312,3280(2 \mathrm{NH}), 1630(\mathrm{C}=\mathrm{O}) \cdot{ }^{13} \mathrm{C}$ NMR $\left(\mathrm{DMSO}-\mathrm{d}_{6}\right)$ : 814.09, 14.31, 22.78, 24.44, 26.19, 29.34, 29.38, 29.68, 29.70, 31.90, 62.47, 93.5, 116.32, 118.79, 129.61, 144.44 . 154.03, 166.66, 169.11. MS $(\mathrm{m} / \mathrm{z}): 501\left(\mathrm{M}^{+1}\right), 500\left(\mathrm{M}^{+}\right)$, 409, 368, 296, 133, 92, 87, 77, 55. For $\mathrm{C}_{29} \mathrm{H}_{47} \mathrm{~N}_{3} \mathrm{O}_{2} \mathrm{~S}(501.34)$ Calcd: C, $69.42 ; \mathrm{H}, 9.44 ; \mathrm{N}, 8.37$; S, 6.39\%. Found: C, 69.44; H, 9.41; N, 8.38; S, 6.38\%.

\section{RESULTS AND DISCUSSION}

3.1 Physical and chemical properties of pomace olive oil

Physical and chemical properties of pomace olive oil are shown in Table 1. The physical examination of oil sample
Table 1 Physical and chemical properties of pomace olive oil.

\begin{tabular}{lrl}
\hline \multicolumn{1}{c}{ Parameters } & Values \\
\hline Refractive index at $25^{\circ} \mathrm{C}$ & & 1.4645 \\
Color at yellow 35 & Red & 5.51 \\
& Blue & 2.90 \\
Conjugated diene $(232 \mathrm{mn})$ & 1.40 \\
Conjugated triene $(270 \mathrm{~nm}$ ) & 0.833 \\
Free fatty acid (\% as oleic acid) & 0.95 \\
Peroxide value (meq. $\mathrm{O}_{2} / \mathrm{kg}$ oil) & 9.51 \\
Iodine value (Hanus) & 80.90 \\
Induction period (hr) & 25.50 \\
\hline
\end{tabular}

includes several analyses of which the most important ones are determination of refractive index and the color. Refractive index is used basically for the estimation of the degree of unsaturation, as well as its correlation with iodine value. The refractive index value at $25^{\circ} \mathrm{C}$ of pomace olive oil was 1.4645. These results were found to be in agreement with Basuny $^{34)}$.

As illustrated in Table 1 the color of pomace olive oil after immediate extraction from pomace olive was yellow cell fixed at 35 and red cell was 5.51 and blue cell was 2.90 respectively. The UV absorbance at 232 and $270 \mathrm{~nm}$ for pomace olive oil was 1.40 and 0.833 respectively. Likewise the free fatty acid ( $\%$ as oleic acid) was $0.95 \%$. The formation of free fatty acid during oxidation as result of cleavage and oxidation of double bonds, this finding in harmony with $\mathrm{Hui}^{35)}$. Besides the peroxide value was 9.51 meq. $\mathrm{O}_{2} / \mathrm{kg}$ oil (Table 1). The IOOC ${ }^{27)}$ and Egyptian Standard of Olive and pomace olive oil ${ }^{36)}$ allow the peroxide value to reach 20 (meq. $\mathrm{O}_{2} / \mathrm{kg}$ oil). These results were found to be in agreement with $\mathrm{Hui}^{35)}$. From the same Table 1 it could be noticed that the iodine value of the pomace olive oil was 80.90. This is to be expected since it may be attributed to the high proportion of unsaturated fatty acids, also the differences between the degree of saturation to unsaturation and total number of double bonds. These results were found to be in agreement with ${ }^{37}$. Data in Table 1 illustrated that the oxidative stability of pomace olive oil. The induction period of pomace olive oil was $25.50 \mathrm{hr}$. The results are agreement with $\mathrm{Ninfali}^{38)}$.

\subsection{Fatty acid composition of pomace olive oil}

Fatty acids composition of the pomace olive oil may differ depending on the variety separation to these fatty acids methyl esters and the determination were carried out by gas -liquid chromatography (GC) in order to identify their types and amounts, the data in Table 2 showed that the fatty acids composition of olive oil extracted from pomace olive oil. From the obtained results it could be ob- 
Table 2 Fatty acid composition of pomace olive oil.

\begin{tabular}{cc}
\hline Fatty acids composition (\%) & Values (\%) \\
\hline C14:0 & 0.04 \\
C16:0 & 16.19 \\
C16:1 & 1.26 \\
C17:0 & 0.00 \\
C17:1 & 0.01 \\
C18:0 & 2.40 \\
C18:1 & 67.37 \\
C18:2 & 11.78 \\
C18:3 & 0.90 \\
Total saturated & 18.68 \\
Total unsaturated & 81.31 \\
\hline
\end{tabular}

served that palmitic acid (C16:0) was the major saturated fatty acids which was $16.19 \%$.

The monounsaturated fatty acids have great importance because of their nutritional implication and effect of oxidative stability of pomace olive oil. Oleic acid (C18:1) was the major monounsaturated fatty acid and was present in higher percentage in pomace olive oil obtained from pomace olive which was represented $67.37 \%$. These results are in agreement with the Egyptian standards of olive oil and pomace olive oil ${ }^{36,39)}$. Concerning linoleic acid (C18:2) which was much more susceptible to oxidation than mono unsaturated fatty acid, the level of linoleic acid in pomace olive oil was $11.78 \%$.

From Table 2 for the other fatty acids palmitoleic (C16:1), stearic (C18:0) and linolenic (C18:3) were found in small amount $1.26 \%, 2.50 \%$ and $0.90 \%$, respectively. As regards the total saturated and unsaturated fatty acids, as illustrated in Table 2, it could be observed that olive oil extracted from pomace olive the total saturated and unsatu- rated was $18.69 \%$ and $81.31 \%$ respectively. These results are in reasonable agreement with ${ }^{28,37)}$.

\subsection{Reactions}

Methyl ester of stearic acid: 1 was used as starting material, and it was prepared by esterification of the corresponding long-chain fatty acid with methanol. Then it was treated with hydrazine hydrate in dry benzene and afforded the corresponding octadecanyl acid hydrazide (2).

The IR spectrum of the later product showed bands at $v$ 3330, 3271-3198, and $1615 \mathrm{~cm}^{-1}$ due to $\mathrm{NH}, \mathrm{NH}_{2}$ and $\mathrm{C}=\mathrm{O}$ functions, respectively. Its mass spectrum revealed a peak at $m / z 297\left(\mathrm{M}^{+}\right)$corresponding to its molecular ion. The ${ }^{1} \mathrm{H}$ NMR spectrum of the same product revealed the appearance of signals due to $\mathrm{NH}$ at $\delta 8.89$ and due to $\mathrm{NH}_{2}$ at $\delta$ 3.51 , in addition to aliphatic protons at $\delta$ 0.9-1.34.

Treatment of fatty acid hydrazide 2 with phenyl isothiocyanate in dry dioxane, gave the corresponding thiosemicarbazide 4 . The IR spectrum of compound 4 revealed a strong absorption band at $v 1658 \mathrm{~cm}^{-1}$ due to $\mathrm{C}=\mathrm{O}$ group and at $1343 \mathrm{~cm}^{-1}$ which indicates the presence of $\mathrm{C}=\mathrm{S}$ group. Its ${ }^{1} \mathrm{H}$ NMR spectrum showed three $\mathrm{D}_{2} \mathrm{O}$-exchangeable signals at $\delta 8.22,4.92$ and 2.54 corresponding to three $\mathrm{NH}$ groups, and a multiplet signal at $\delta 7.20-7.64$ due to aromatic protons, in addition to a multiplet at $\delta$ 0.88-1.36 corresponding to aliphatic protons.

thiosemicarbazide 4 undergoes intramolecular cyclization in basic medium, to give the $s$-triazole derivative 5 (Scheme 3). The structure of the product was established by its elemental analysis and spectral data, For instance, its IR spectrum showed a strong absorption band at 1345 $\mathrm{cm}^{-1}$ due to $\mathrm{C}=\mathrm{S}$ group. The ${ }^{1} \mathrm{H}$ NMR spectrum of the same compound reveled $\mathrm{D}_{2} \mathrm{O}$-exchangble signal at $\delta 8.04$ due to SH proton and multiplate signal at 7.21-7.64 due to aromatic protons, in addition to aliphatic protons at $\delta 0.9$ 1.36 .

Methylation of the triazolthione 5 with methyl iodide af-

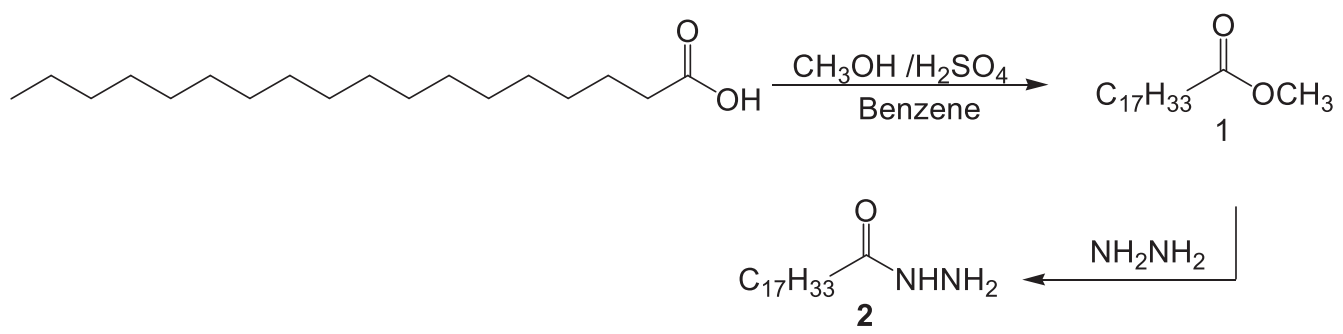

Scheme 1

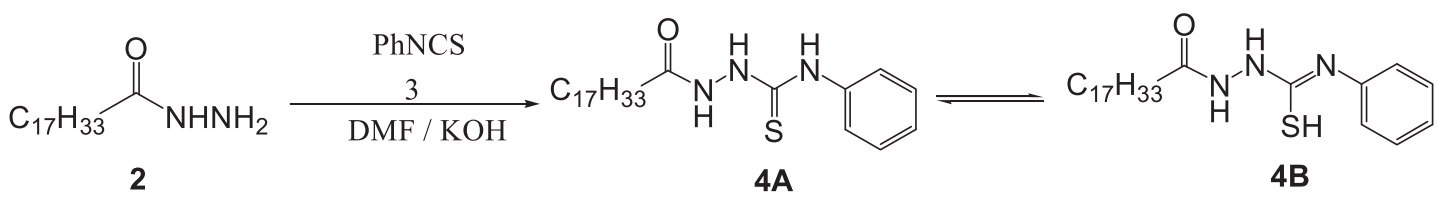

Scheme 2 
forded only one product identified as 3-heptadecanyl-5(methylthio)-4-phenyl-4H-1,3,4-triazole (6) (Scheme 3). The structure of compound 6 was confirmed on the basis of its elemental analysis and spectral data. For example, its IR spectrum showed the disappearance of $\mathrm{C}=\mathrm{S}$ absorption band, and its ${ }^{1} \mathrm{H}$ NMR spectrum revealed a mutiplate signal at $\delta$ 7.21-7.55 due to aromatic protons, and a signal at $\delta$ 3.44 due to $\mathrm{CH}_{3}$ protons, in addition to aliphatic protons signal at $\delta$ 0.92-1.33.

When compound 6 was treated with hydrazine hydrate, it afforded the corresponding 1-(5-heptadecanyl-4-phenyl-4H-1,2,4-triazol-3-yl) hydrazine (7) (Scheme 3). The IR spectrum of the product 7 exhibited strong absorption bands at n 3320 and $3220-3197 \mathrm{~cm}^{-1}$ corresponding to hy- drazino group, It's ${ }^{1} \mathrm{H}$ NMR showed disappearance of $\mathrm{CH}_{3}$ signal, and it revealed signal due to $\mathrm{NH}$ at $\delta 4.13$ and at $\delta$ 2.70 due to $\mathrm{NH}_{2}$ protons, in addition to aliphatic protons signal at $\delta 0.89-1.33$.

Intramolecular cyclization of the thiosemicarbazide 4 in acid medium, afforded the corresponding thiadiazole derivative 8 . The IR spectrum of compound 8 showed an absorption band at $3595 \mathrm{~cm}^{-1}$ which indicate the presence of $\mathrm{NH}$ group. Also, it's ${ }^{1} \mathrm{H}$ NMR revealed a signal at $\delta 9.73$ $\left(\mathrm{D}_{2} \mathrm{O}\right.$-exchangeable) due to $\mathrm{NH}$ proton, in addition to aliphatic protons at $\delta$ 0.91-1.33.

When the thiosemicarbazide 4 was treated with ethyl chloro (arylhydrazono) acetate derivatives 9a-b in dimethylformamide, and in the presence of triethylamine, it fur-



5A

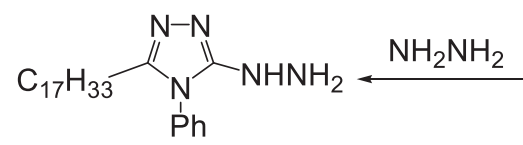

7

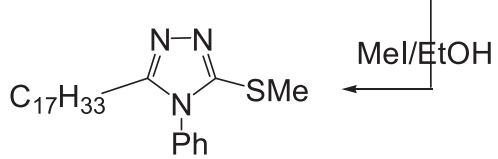
$\mathrm{Ph}$

\section{Scheme 3}

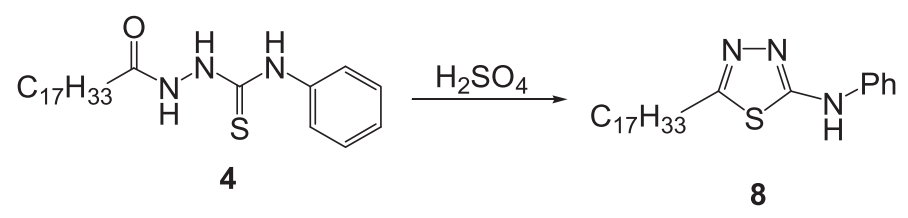

Scheme 4

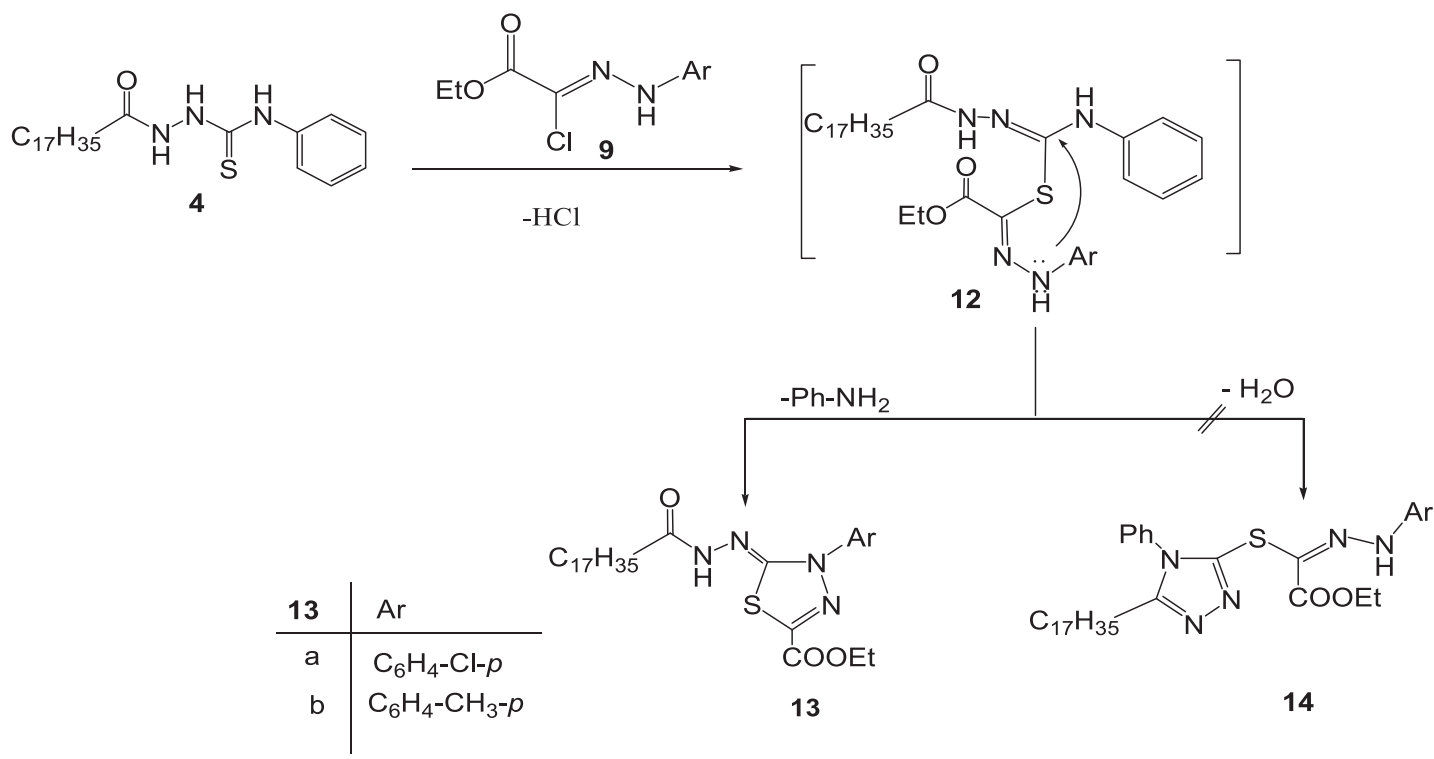

Scheme 5 


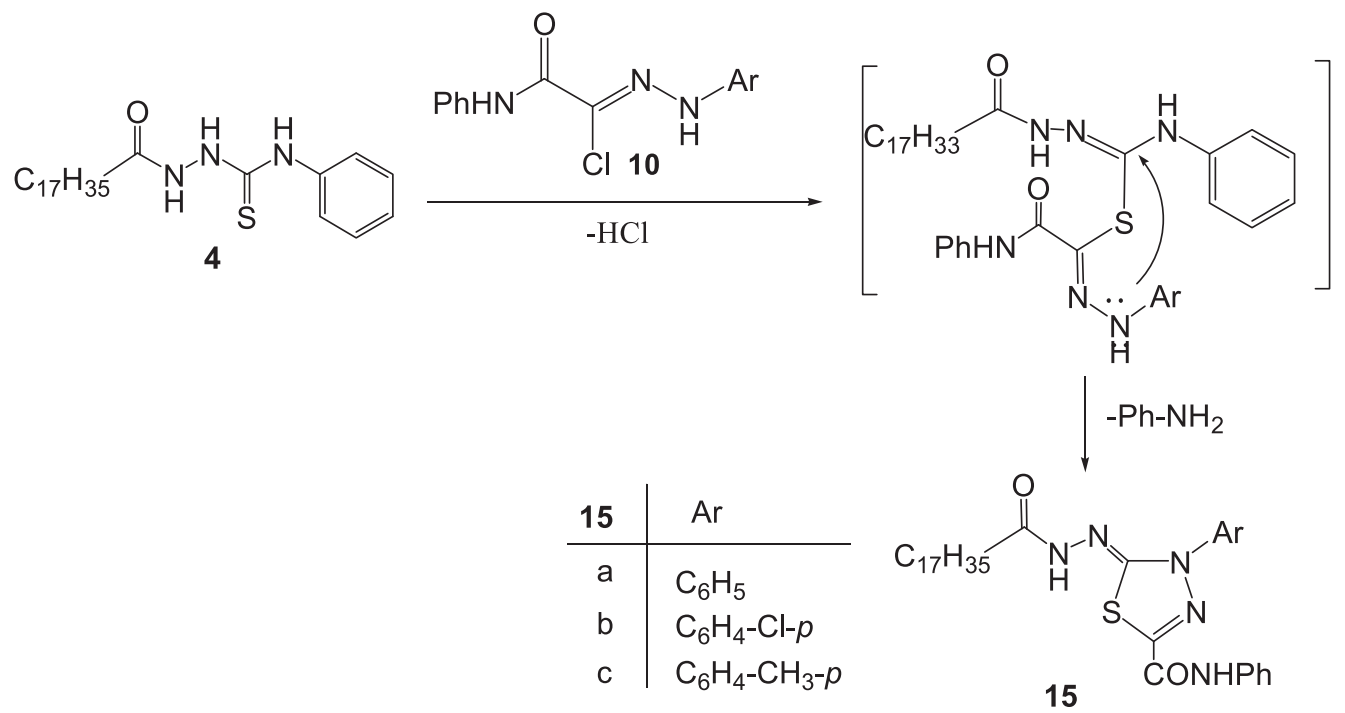

Scheme 6

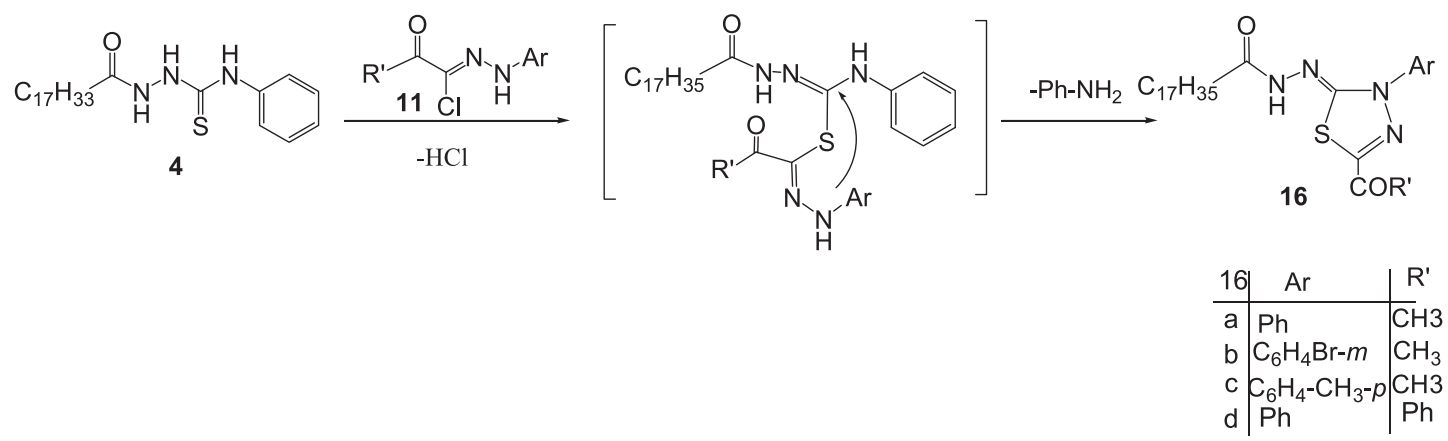

Scheme 7

nished a single product for which the two possible structures 13 and 14 can be drawn (Scheme 5). However, elemental analysis and spectral data were in complete agreement with the thiadiazole structure 13 . The ${ }^{1} \mathrm{H}$ NMR spectrum of compound 13a, taken as a typical example, revealed signals at $\delta 8.00\left(\mathrm{D}_{2} \mathrm{O}\right.$-exchangable) corresponding to the NH proton, and at $\delta$ 7.1-7.3 due to aromatic protons, in addition to aliphatic protons signal at $\delta$ 0.91-1.33. The IR spectrum of the same compound exhibited a characteristic band for $\mathrm{NH}$ absorption at $v 3365 \mathrm{~cm}^{-1}$ in addition to the two carbonyl absorption bands at $v 1746-1667 \mathrm{~cm}^{-1}$. The formation of compounds $13 a-b$ is assumed to take place via the alkylation of the thiosemicarbazide 4 to afford the acyclic non-isolable intermediates 12a-b which underwent intramolecular cyclization through the elimination of aniline molecule to afford the substituted thiadiazole derivatives 13a-b as shown in Scheme 5.

Similarly, when the thiosemicarbazide 4 was treated with the phenylcarbamoylarylhydrazonyl chloride 10a-c, under the same experimental condition, it afforded (3-Aryl- $N-5$ (phenylcarbamoyl)-1,3,4-thiadiazol-2 (3H) -ylidene) octadecanehydrazide 15a-c (Scheme 6). The structures of the formed compounds 15a-c were confirmed on the basis of their elemental analysis and spectral data (see Experimental part).

The thiosemicarbazide 4 reacts also with 2-oxo- $N$-arylpropanehydrazonoyl chlorides $11 \mathrm{a}-\mathrm{c}$ and $N$-phenylbenzohydrazonoyl chloride $11 \mathrm{~d}$, in DMF, and in the presence of triethylamine, to give the corresponding thiadiazole derivatives 16a-d as shown in Scheme 7. The ${ }^{1} \mathrm{H}$ NMR spectrum of compound 16a, taken as a typical example of the series, revealed signals at $\delta 7.89\left(\mathrm{D}_{2} \mathrm{O}\right.$-exchangble $)$ due to the amino proton, and at d 7.1-7.4 due to phenyl protons, in addition to aliphatic protons multiplet at $\delta 0.89-1.33$. The IR spectrum of $16 \mathrm{a}$ showed a characteristic band for $\mathrm{NH}$ absorption band at $v 3371 \mathrm{~cm}^{-1}$, in addition to two $\mathrm{C}=\mathrm{O}$ absorption bands at $v 1740$ and $1666 \mathrm{~cm}^{-1}$.

Treatment of a solution of the thiosemicarbazide 4 , in ethanol with the haloketones $17 \mathrm{a}-\mathrm{c}$ in the presence of triethylamine, afforded in each case, only one isolated product for which the two possible structures 19 and 20 can be considered. Elemental analysis and spectral data of the reaction products were compatible only with the thiadiazine derivatives 20a-c, as shown in Scheme 9. The ${ }^{1} \mathrm{H}$ 


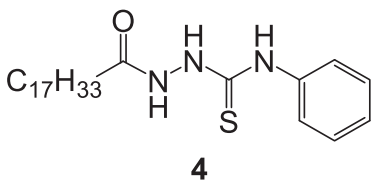<smiles>O=C(Br)CCCCCC(=O)NNC(Nc1ccccc1)C(=O)Br</smiles>

\begin{tabular}{r|l}
20 & $\mathrm{Ar}$ \\
\hline $\mathrm{a}$ & $\mathrm{C}_{6} \mathrm{H}_{5}$ \\
b & $\mathrm{C}_{6} \mathrm{H}_{4}-\mathrm{Cl}-p$ \\
c & $\mathrm{C}_{6} \mathrm{H}_{4}-\mathrm{CH}_{3}-p$
\end{tabular}

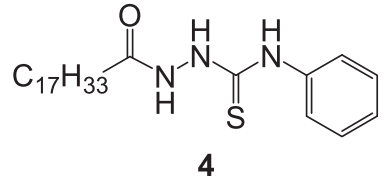<smiles>[R]C(=O)CCl</smiles><smiles>[R]C(=O)C1=C(N)NN=C(Nc2ccccc2)S1</smiles>

22

$22 R^{2}$

a $\mathrm{CH}_{3}$

b) $\mathrm{OCH}_{2} \mathrm{CH}_{3}$

\section{Scheme 9}

NMR spectrum of compound 20a for example, revealed signals at $\delta 8.81$ and 4.42 due to the two NH protons, in addition to an aliphatic protons multiplate signal at $\delta 0.91$ 1.35. The IR spectrum of the same compound showed two $\mathrm{NH}$ absorption bands at 3384 and $3312 \mathrm{~cm}^{-1}$, in addition to $\mathrm{C}=\mathrm{O}$ absorption band at $v 1637 \mathrm{~cm}^{-1}$. The reaction of 4 with haloketones was assumed to take place via the non isolable acyclic intermediates 18a-c that underwent intramolecular cyclization through the elimination of water molecule and afforded the corresponding thiadiazine derivatives 20a-c (Scheme 8).

Analogously, the thiosemicarbazide 4 was reacted with $\alpha$-haloketone $21 \mathrm{a}-\mathrm{b}$ and afforded the corresponding products 22a-b (Scheme 9). The structures of the isolated products were confirmed according to their elemental analysis and spectral data (see Experimental part).

\section{Antimicrobial Activity:}

The compounds $(100 \mu \mathrm{g} / \mathrm{mL})$ were tested in vitro for antibacterial activity against four bacterial species (two gram positive and two gram negative) namely Staphylococcus
Epiderm, Enterococus fesalis, Enterobacter cloacae, and Flavo-Bacterium, using disk diffusion method ${ }^{40)}$, for the antibacterial activity of each compound in diethyl ether as solvent.

Inhibition zone diameter(IZD), in mm/mg compound tested was taken as the criterion for antimicrobial activity. The antibiotics Vacomycin - rifampin, Penicillin G, Gentamycin - cephpalosporin - cefotaxime Imipenem and Normal flora Natpathegen, were used as references to evaluate the potency of the tested compounds under the same condition.

The results are depicted in the following table, on the following basis:

- The solvent used was diethyl ether.

- Concentration of the sample in $100 \mu \mathrm{g} / \mathrm{mL}$.

- $\mathrm{IZD}=2-10 \mathrm{~mm}$ beyond control $=+$ (low activity $)$.

- $\mathrm{IZD}=11-24 \mathrm{~mm}$ beyond control $=++$ (moderate activity).

- IZD $=25-35 \mathrm{~mm}$ beyond control $=+++$ (high activity).

By comparing the results from Table 3 to that obtained from pervious study ${ }^{25}$, which was carried out on the same hetero cyclic compounds but those were attached to octa- 
Table 3 Antimicrobial activity of the tested compounds.

\begin{tabular}{|c|c|c|c|c|}
\hline \multirow{3}{*}{ Compounds No. } & \multicolumn{4}{|c|}{$\begin{array}{l}\text { IINHIBITION ZONE DIAMETER (IZD) } \\
(\mathrm{MM} / \mathrm{MG} \text { COMPOUND TESTED) I }\end{array}$} \\
\hline & \multicolumn{2}{|c|}{ Gram + ve } & \multicolumn{2}{|c|}{ Gram-ve } \\
\hline & $\begin{array}{l}\text { Staphylococcus } \\
\text { Epiderm }\end{array}$ & $\begin{array}{l}\text { Enterococus } \\
\text { fesalis }\end{array}$ & $\begin{array}{l}\text { Enterobacter. } \\
\text { cloacae }\end{array}$ & Flavobacterium \\
\hline CONTROL & 0.0 & 0.0 & 0.0 & 0.0 \\
\hline 4 & + ve & $++\mathrm{ve}$ & - & $+++\mathrm{ve}$ \\
\hline 5 & ++ ve & + ve & $+\mathrm{ve}$ & - \\
\hline 6 & $++\mathrm{ve}$ & ++ ve & ++ ve & $+\mathrm{ve}$ \\
\hline 7 & ++ ve & ++ ve & + ve & + ve \\
\hline $13 \mathrm{a}$ & - & $++\mathrm{ve}$ & $++\mathrm{ve}$ & $++\mathrm{ve}$ \\
\hline $13 b$ & ++ ve & + ve & $++\mathrm{ve}$ & ++ ve \\
\hline $15 \mathrm{a}$ & $++\mathrm{ve}$ & + ve & $+\mathrm{ve}$ & $+\mathrm{ve}$ \\
\hline $15 b$ & $++\mathrm{ve}$ & $+++\mathrm{ve}$ & $++\mathrm{ve}$ & + ve \\
\hline $15 c$ & ++ ve & ++ ve & ++ ve & ++ ve \\
\hline $16 \mathrm{a}$ & - & $++\mathrm{ve}$ & $++\mathrm{ve}$ & + ve \\
\hline $16 b$ & $+\mathrm{ve}$ & $++\mathrm{ve}+$ & $++\mathrm{ve}$ & + ve \\
\hline $16 \mathrm{c}$ & $+\mathrm{ve}$ & $++\mathrm{ve}$ & $++\mathrm{ve}$ & $++\mathrm{ve}$ \\
\hline $16 \mathrm{~d}$ & ++ ve & ++ ve & ++ ve & + ve \\
\hline $20 \mathrm{a}$ & ++ ve & ++ ve & + ve & + ve \\
\hline $20 b$ & ++ ve & ++ ve & ++ ve & ++ ve \\
\hline $20 c$ & ++ ve & - & $++\mathrm{ve}$ & - \\
\hline $22 \mathrm{a}$ & ++ ve & + ve & - & $++\mathrm{ve}$ \\
\hline $22 b$ & ++ ve & $+++\mathrm{ve}$ & ++ ve & ++ ve \\
\hline References & $\begin{array}{l}\text { Vacomycin - } \\
\text { rifampin }\end{array}$ & Penicillin G & $\begin{array}{c}\text { Gentamycin - } \\
\text { cephpalosporin } \\
\text { - cefotaxime } \\
\text { Imipenem }\end{array}$ & $\begin{array}{l}\text { Normal flora } \\
\text { Natpathegen }\end{array}$ \\
\hline
\end{tabular}

decenyl instead of octadecanyl moiety and on the same bacterial species, it's found that, antimicrobial activity of hetero cyclic compounds attached to octadecenyl was slightly higher than that attached to octadecanyl. Thus, the presence of unsaturated bond in the fatty moiety increases the antimicrobial activity of the compound.

\section{CONCLUSION}

The new prepared compounds are expected to be biologically beneficial in combating microorganisms, moreover these compounds are expected to have several advantages over the parent heterocyclic one, where they may easily penetrate the lipoprotein cell membrane, they may be stored in liver and skin as a lipid component that can be used whenever they needed, also the toxicity and side effects of this new compounds may be reduced by the presence of the long chain fatty acid residue which circulate the hetero nucleus.

\section{ACKNOWLEDGMENT}

Authors wish to thank to Prof. Dr. Mohamoud El Mallah Professor of fats and oils technology, Fats and Oils Department, National Research Center (NRC), Cairo, Egypt for his helpful guidance, valuable criticism and fruitful advice.

Authors are also grateful to Prof. Dr. Feryal Zaher, Professor of fats and oils technology, Fats and Oils Department, National Research Center(NRC), Cairo, Egypt, for her valuable advice. 


\section{REFERENCES}

1) Sonntag, N. O. V.; Johnson, R. W.; Fritz, E. Fatty Acids in Industry. pp. 23-72, Marcel Dekker, Inc., New York (1988).

2) Maheshwari, P.; Nikolov, Z. L.; White, T. M.; Hartel, R. Solubility of fatty acids in Supercritical carbon dioxide. J. Am. Oil Chem. Soc. 69, 1069-1076(1992).

3) Rauf, A.; Sharma, S.; Gangal, S. Microwave assisted efficient one-pot synthesis of 3,5,6-trisubstituted-1,2,4triazines from fatty acid hydrazides under solvent-free conditions and their antimicrobial activity. ARKIVOC 16, 137-147 (2007).

4) Ghorab, M. M.; El-Sharief, S.; Ammar, A. M.; Mohamed, I. Synthesis and antifungal activity of some new miscellaneous s-triazoles. Phosphorus, Sulfur Silicon. Relat. Elem. 173, 223-233(2001).

5) Wang, Z.; Shi, H.; Shi, H. J. Synthesis of chiral fused heterocyclic compounds containing 1,2,4-triazolering. Heterocycl. Chem. 38, 355-357 (2001).

6) Palaska, E.; Sahin, G.; Kelicen, P.; Durlu, N. T.; Altinok, G. Synthesis and anti-inflammatory activity of 1-acylthiosemicarbazides, 1,3,4-oxadiazoles, 1,3,4-thiadiazoles and 1,2,4-triazole-3-thiones. Farmaco 57, 101107 (2002).

7) Labanauskas, L.; Kalcas, V.; Udrenaite, E.; Gaidelis, P.; Brukstus, A.; Dauksas, V. Synthesis of 3-(3,4-dimethoxyphenyl) -1 H-1,2,4-triazole-5-thiol and 2-amino-5-(3,4-dimethoxyphenyl) -1,3,4-thiadiazole derivatives exhibiting anti-inflammatory activity. Pharmazie 56, 617-619 (2001).

8) Onkol, T.; Cakir, B.; Sahin, M. F. Synthesis and antinociceptive activity of 2-[(2-oxobenzothiazoline-3-yl) methyl]-5-amino alkyl/aryl-1,3,4-thiadiazole. Turk. J. Chem. 28, 461-468(2004).

9) Schenone, S.; Bruno, O.; Banise, A.; Bondavalli, F.; Filippeli, W.; Falcone, G.; Giardano, L.; Vitelli, M. R. Bioorg. Med. Chem. 9, 2149-2153(2001).

10) Gokce, M.; Cakir, B.; Erol, K.; Sahin, M. F. Synthesis and antinociceptive activity of [ (2-oxobenzothiazolin3-yl) methyl]-4-alkyl/aryl-1,2,4-triazoline-5-thiones. Arch. Pharm. 334, 279-283 (2001).

11) Baldwin, J. J.; Engelhardt, E. L.; Hirschmann, R.; Ponticella, G. S.; Atkinson, J. G.; Wasson, B. K.; Sweet, C. S.; Scriabini, A. Scriabini, Heterocyclic analogues of the antihypertensive beta-adrenergic blocking agent (S) -2-[3-(ter-butylamino)-2-hydroxypropoxy]-3-cyanopyridine. J. Med. Chem. 23, 65-70 (1980).

12) Varvaresou, A.; Tsantili-Kakoulidou, A.; Siatra-Papastasikoudi, T.; Tiligada, E. Synthesis and biological evaluation of indole containing derivatives of thiosemicarbazide and their cyclic 1,2,4-triazole and 1,3,4-thiadiazole analogs. Arzneimittelforschung 50, 48-54 (2000).

13) Foroumadi, A.; Mirzaei, M.; Shafiee, A. Synthesis and antituberculosis activity of 2-aryl-1,3,4-thiadiazole derivatives. Pharmazie 56, 610-612(2001).

14) Mamola, M. G.; Falagiani, V.; Zanpieir, D.; Vio, L.; Banfi, F. Synthesis and antimycobacterial activity of [5(pyridin-2-yl)-1,3,4-thiadiazol-2-ylthio] acetic acid arylidene-hydrazide derivatives. Farmaco 56, 587-592 (2001).

15) Chen, H.; Li, Z.; Han, Y. Synthesis and fungicidal activity against Rhizoctonia solani of 2-alkyl (Alkylthio) -5-pyrazolyl-1,3,4-oxadiazoles (Thiadiazoles). J. Agric. Food Chem. 48, 5312-5315(2000).

16) Zou, X. J.; Jin, G. Y.; Zang, Z. X. Synthesis, fungicidal activity, and QSAR of pyridazinonethiadiazoles. J. Agric. Food Chem. 50, 1451-1454(2002).

17) Zou, X. J.; Lai, L. H.; Jin, G. Y.; Zhang, Z. K. Synthesis, fungicidal activity, and 3D-QSAR of pyridazinone-substituted 1,3,4-oxadiazoles and 1,3,4-thiadiazoles. J. Agric. Food Chem. 50, 3757-3760 (2002).

18) Dong, X. G.; Yan, L.; Song, X. J.; Du, Y. X. Synthesis and antimicrobial activity of N-[5-(3-pyridyl)-1, 3, 4-thiadiazol-2-yl] -N'-aroyl urea. Yao Xиe Xue Bao. 42, 108-110 (2007).

19) Clerici, F.; Pocav, D.; Guido, M.; Lochi, A.; Perline, V.; Brufani, M. Synthesis of 2-amino-5-sulfanyl-1,3,4-thiadiazole derivatives and evaluation of their antidepressant and anxiolytic activity. J. Med. Chem. 44, 931936 (2000).

20) Rauf, A.; Parveen, H. characterization and antimicrobial activity of fatty alkenoates. Ind. J. Chem. 44B, 1273-1276 (2005).

21) Rauf, A.; Banday, M. R.; Matto, R. M. Synthesis, Characterization and Antimicrobial Activity of Long-Chain Hydrazones. Acta Chim. Slov. 55, 448-452 (2008).

22) Ahmed, S. M.; Ahmad, F.; Osman, S. M. Preparation and characterization of derivatives of isoricinoleic acid and their antimicrobial activity. J. Am. Oil Chem. Soc. 62, 1578-1580 (1985).

23) Khan, M. W. Y.; Ahmad, F.; Ahmad, I.; Osman, S. M. Nonedible seed oils as insect repellant. J. Am. Oil Chem. Soc. 60, 949-950(1983).

24) Mujeebur-Rahman, V. P.; Mukhtar, S.; Ansari, W. H.; Lemiere, G. Synthesis, stereochemistry and biological activity of some novel long alkyl chain substituted thiazolidin-4-ones and thiazan-4-one from 10-undecenoic acid hydrazide. Eur. J. Med. Chem. 40, 173-184 (2005).

25) Soliman, H. M. Synthesis of Some Fat Based Heterocyclic from Olive Oil Production Waste Precursors. Ph. D. Thesis, Chemistry Department College of Sciences, Cairo University (2010).

26) A. O. A. C. Association of Official Agriculture Chemists. Official Methods of Analysis of $18^{\text {th }}$ ed., D. C. USA (2005).

27) International olive oil council(IOOC) Trade standard 
applying to olive oil and olive pomace oil. COI/T. 15. Nc No.3/Rev.4. November (2009).

28) Hegarty, A. F.; Cashman, M. P.; Scott, F. L. Mechanism of 1,3-dipolar ion formation. Chem. Commun. 13, 684-685 (1971).

29) Girls, K.; Edwin, G. Chem. Abstr. 72, 54978e(1970).

30) Shawali, A. S.; Osman, A. Synthesis and reactions of phenylcarbamoylarylhydrazidic chlorides. Tetrahedron 27, 2517-2528(1971).

31) Dieckmann, W.; Platz, O. Ueber eine neue Bildungsweise von Osotetrazonen. Chem. Ber. 38, 2989-2990 (1960).

32) Cowper, R. M.; Daviadson, L. H. PHENACYL BROMIDE [Acetophenone, $\alpha$-bromo-]Organic Synthesis Coll. 2, 480-481 (1943).

33) Wolkoff, P. A New Method of Preparing Hydrazonyl Halides. Can. J. Chem. 53, 1333-1335 (1975).

34) Basuny, M. M. A. Use of natural antioxidants for increase stability of sunflower oil. Ph. D. Thesis, Faculty of Agriculture, Cairo University (2000).
35) Hui, Y.H. Baileys' industrial oil and fats products (Fifth Edition, Vol. 2 and 4) John Wiley Sons. Inc., New York (1978).

36) Egyptian Standard Organization, Vegetable oils standard, olive oils and olive pomace oils (No, 49/2), Egyptian Organization for standardization (2005).

37) Yap, P. H.; Man, J. M.; De Man, L. Polymorphism of palm oil and palm oil products. J. Am. Oil Chem. Soc. 66, 693-697 (1989).

38) Ninfali, P.; Aluigi, G.; Bacchiocca, M.; Magnani, M. Antioxidant capacity of extra virgin olive oils. J. Am. Oil Chem. Soc. 78, 243-247(2001).

39) Manai, H.; Mahjoub-Haddada, F.; Oueslati, I.; Daoud, D.; Zarrouk, M. Characterization of monovarietal virgin olive oils from six crossing varieties. Scientia Horticultrae 115, 252-260 (2008).

40) Gould, J.; Bowie, J. H. The determination of bacterial sensitivity to antibiotics. Edinb. Med. J. 59, 178-199 (1952). 